\title{
Symmetry breaking by bifundamentals
}

\author{
A. N. Schellekens \\ Nikhef Theory Group, Science Park 105, 1098 XG Amsterdam, The Netherlands \\ and Instituto de Física Fundamental, CSIC, Serrano 123, Madrid 28006, Spain
}

(Received 22 November 2017; published 12 March 2018)

\begin{abstract}
We derive all possible symmetry breaking patterns for all possible Higgs fields that can occur in intersecting brane models: bifundamentals and rank-2 tensors. This is a field-theoretic problem that was already partially solved in 1973 by Ling-Fong Li [1]. In that paper the solution was given for rank-2 tensors of orthogonal and unitary group, and $U(N) \times U(M)$ and $O(N) \times O(M)$ bifundamentals. We extend this first of all to symplectic groups. When formulated correctly, this turns out to be straightforward generalization of the previous results from real and complex numbers to quaternions. The extension to mixed bifundamentals is more challenging and interesting. The scalar potential has up to six real parameters. Its minima or saddle points are described by block-diagonal matrices built out of $K$ blocks of size $p \times q$. Here $p=q=1$ for the solutions of Ling-Fong $\mathrm{Li}$, and the number of possibilities for $p \times q$ is equal to the number of real parameters in the potential, minus 1 . The maximum block size is $p \times q=2 \times 4$. Different blocks cannot be combined, and the true minimum occurs for one choice of basic block, and for either $K=1$ or $K$ maximal, depending on the parameter values.
\end{abstract}

DOI: $10.1103 /$ PhysRevD.97.056007

\section{INTRODUCTION}

The purpose of this paper is to complete the classic work of Ling-Fong Li [1] (see also [2] for corrections), in which symmetry breaking patterns caused by Higgs fields in various representations of various groups are considered. Perhaps surprisingly, there does not exist a general formula or algorithm that deals with this group-theoretical problem in full generality, quite unlike computing the tensor product for arbitrary representations, for example. In [1] some special cases were selected based on what seemed interesting at that time. With remarkable foresight, the author considered representations that can occur as massless states in open string models, or more precisely intersection brane models: rank-2 tensors and bifundamentals. However, the results of [1] do not cover all possible brane configurations: symplectic groups were not discussed, and neither were mixed group types: unitary orthogonal, unitary symplectic, and orthogonal symplectic. Here we complete these results by working out the missing ones.

In field theory any study of Higgs symmetry breaking is inevitably limited to an infinitesimal subset of the allowed Higgs representations. But in string theory those representations are limited to the ones that can be massless. In

Published by the American Physical Society under the terms of the Creative Commons Attribution 4.0 International license. Further distribution of this work must maintain attribution to the author(s) and the published article's title, journal citation, and DOI. Funded by SCOAP ${ }^{3}$. intersecting brane models, rank-2 tensors and bifundamentals are the only representations that can occur. So one can actually solve the problem in full generality, if there is only a single Higgs field.

The concrete reason for embarking on this study originates from attempts to generalize a previous paper [3]. In that paper a surprisingly successful attempt was made to derive the standard model from requirements on the complexity of the low-energy physics it produces. This is the opposite of trying to understand the standard model from some high-energy symmetry principle, as one usually does. In a limited set of two-stack brane models, the $\mathrm{N}$-family standard model turned out to stand out clearly as the case with the richest kind of "atomic physics." This may be viewed as a concretely defined proxy for an anthropic argument. A crucial role was played by the assumption that a single Higgs field renders the entire spectrum nonchiral.

When we attempted to extend this analysis to multistack models, we ran into a rather stubborn problem. The generalization required considering Higgs fields in any representation allowed by the brane configurations. Since branes (or open strings) can have unitary, orthogonal, and symplectic groups (U, O, and $\mathrm{S}$ for short) [4], and since bifundamentals can arise from any combination of branes, we needed the aforementioned generalization of [1]. Although in some cases educated guesses can be made based on group theory arguments, this is not satisfactory.

While studying this problem it became clear that the general case is substantially more complicated than the 
U-U and O-O cases studied in [1]. Instead of two terms in the quartic potential for $\mathrm{U}-\mathrm{U}, \mathrm{O}-\mathrm{O}$, and S-S, one gets three for U-O and U-S, and five for O-S, with up to six real coupling constants. Although initially this seemed intractable, we found that it can in fact be solved completely and exactly, and that it even simplifies the original analysis of Ling-Fong Li. In fact, a rather beautiful solution emerges.

Although the inspiration for this work came from string theory, all of it is in fact classical field theory. But there is a remnant of the stringy origin, namely the fact that we use the full unitary group and not the simple group $S U(N)$, because $U(N)$ is what comes naturally out of string theory. One can get $S U(N)$ in open string theory in four dimensions if the $U(1)$ phase symmetry is broken by axion mixing, but this is an entirely separate and modeldependent issue that we do not consider here. Furthermore not only is the full gauge group of the theory a product of $U(N), O(N)$, and $U S p(N)$ factors, but we find that the same is true for the stabilizer of the Higgs vacuum, the broken subgroup. We find that $S U(N)$ groups occur only as part of a $U(N)$, or as $U S p(N)$ for the special case $N=2$, because $U S p(2)$ is isomorphic to $S U(2)$. This suggests that the broken subgroups may have a simply string theory interpretation. Indeed, this hints at an interpretation in terms of a phenomenon known as "brane recombination," discussed for example in [5]. This is an intriguing possibility, but we do not explore this further in the present work, since this lies beyond the scope of field theory.

This paper has two parts. In the first part we discuss the most interesting case, namely, bifundamentals between different branes. The second part is about self-intersections, or in other words rank-2 tensors. Here the only cases not yet considered in [1] are (anti)-symmetric tensors of symplectic groups. The breaking patterns and energy considerations turn out to be a natural extension of those for unitary and orthogonal groups. Besides symplectic rank-2 tensors the other novelty in this part is a greatly simplified derivation, made possible by some fairly old but not widely known theorems on matrix (skew) diagonalization.

For readers who just need the results and who are not interested in the methods and derivations, two summary sections are provided, namely, IIG for bifundamentals and IIIC for rank-2 tensors.

\section{BIFUNDAMENTALS}

We consider a Higgs field in a bifundamental representation of groups $G(N) \times H(M)$, where each group can be orthogonal, unitary, or symplectic. The global groups are $G(N)=O(N), U(N)$, or $U S p(N)$ and analogously for $H(M)$. The precise definition of $U S p(N)$ is given in the Appendix. The symplectic groups only exist for even matrix dimensions, but to keep the notation universal we use $N$ (or $M$ ) in all cases.

\section{A. The potential}

\section{Invariant contractions}

Consider a Higgs field $\phi_{i \alpha}$ in a bifundamental representation of groups $G(N) \times H(M)$, with $i=1, \ldots, M$ and $\alpha=1, \ldots, N$. We consider a renormalizable Higgs potential. This implies that it can only have quadratic and quartic terms. Cubic terms are not possible with bifundamentals.

Since all groups are embedded in a unitary group, one always obtains invariance under the left or right algebra if $\phi$ is combined with $\phi^{*}$, and the left and right indices are contracted. This can be done in only one way for quadratic terms, and in two ways for quartic terms.

In addition, depending on the algebra one considers, it may be possible to obtain gauge invariant combinations by contracting indices of two $\phi$ 's (or two $\phi^{*}$ 's) with each other, provided an invariant contraction matrix exists. We denote the contraction matrix for the first index as $D_{i j}$, and the one for the second index as $C_{\alpha \beta}$. In a suitable basis, these matrices can be chosen as either a Kronecker $\delta$ for orthogonal groups, or a skew-diagonal matrix with diagonal $i \sigma_{2}$ blocks for symplectic groups. The latter is denoted $\Omega$. One can of course define other bases, and, in particular, for symplectic groups $U S p(2 K)$ there are two that are often used. One is the matrix $\Omega$, which can be written as $\mathbb{1}_{K} \otimes\left(i \sigma_{2}\right)$, and the other canonical choice is $\left(i \sigma_{2}\right) \otimes \mathbb{1}_{K}$; this is the matrix $h_{A}$ shown in Eq. (A2). Of course no results depend on that choice, but the form $\Omega$ is convenient in some computations, and also makes a formulation in terms of quaternions possible in certain cases.

We define

$$
\begin{aligned}
& C^{T}=\epsilon_{C} C, \\
& D^{T}=\epsilon_{D} D,
\end{aligned}
$$

where $\epsilon_{C}$ and $\epsilon_{D}$ are signs; they are +1 if $C$ or $D$ are Kronecker $\delta$ 's, and -1 if $C$ or $D$ is equal to a matrix $\Omega$ (or $h_{A}$ ).

\section{Mass terms and reality conditions}

If both $C$ and $D$ exist, we can write down two distinct mass terms,

$$
\begin{aligned}
\phi_{i \alpha} \phi_{i \alpha}^{*} & =\operatorname{Tr} \phi \phi^{\dagger}, \\
\phi_{i \alpha} \phi_{j \beta} D_{i j} C_{\alpha \beta} & =\operatorname{Tr} \phi C \phi^{T} D .
\end{aligned}
$$

The second one vanishes unless $\epsilon_{C}=\epsilon_{D}$, so it exists only if both groups are orthogonal or both groups are symplectic. The existence of an additional mass term indicates that there are two separate fields rather than just one. We can eliminate one of these components by imposing a reality condition

$$
\phi^{*}=D^{T} \phi C
$$


With the canonical choices for $C$ and $D$ given above, these reality conditions simply imply that $\phi$ is real or quaternionic for orthogonal or symplectic groups respectively (see the Appendix for more details). In the latter case this means that $\phi$ can be written in terms of quaternions, defined on the $2 \times 2$ blocks of the matrices $\Omega_{i j}$ and $\Omega_{\alpha \beta}$. Both kinds of reality conditions imply a reduction by a factor of 2 in the number of degrees of freedom. A general complex field may be written as a real part plus an imaginary field; the quaternionic analog is that its $2 \times 2$ block can be written as a $q+i q^{\prime}$, where $q$ and $q^{\prime}$ are quaternions.

\section{Quartic terms}

If both $C$ and $D$ exist, the most general invariant potential has the form

$$
\begin{aligned}
V= & -\mu^{2} \operatorname{Tr} \phi \phi^{\dagger}+\frac{1}{2} \lambda_{1}\left(\operatorname{Tr} \phi \phi^{\dagger}\right)^{2}+\frac{1}{2} \lambda_{2} \operatorname{Tr}\left(\phi \phi^{\dagger}\right)^{2} \\
& +\frac{1}{2} \lambda_{3} \operatorname{Tr}\left(\phi C \phi^{T}\right)\left(\phi C \phi^{T}\right)^{\dagger}+\frac{1}{2} \lambda_{4} \operatorname{Tr}\left(\phi^{T} D \phi\right)\left(\phi^{T} D \phi\right)^{\dagger} \\
& +\frac{1}{4}\left[\lambda_{5} \operatorname{Tr}\left(\phi C \phi^{T} D\right)^{2}+\text { H.c. }\right] .
\end{aligned}
$$

The normalization of this potential differs from the one used in [1]. We have chosen a normalization so that for complex fields all numerical factors in the equation of motion are equal to 1 . For this reason our potential is larger by an overall factor 2 with respect to [1]. Since we are only interested in the extrema, this is irrelevant. For future purposes we write the potential as

$$
\begin{aligned}
V= & -\mu^{2} \phi_{i \alpha} \phi_{i \alpha}^{*}+\frac{1}{2} \lambda_{1} V_{1}+\frac{1}{2} \lambda_{2} V_{2}+\frac{1}{2} \lambda_{3} V_{3}+\frac{1}{2} \lambda_{4} V_{4} \\
& +\frac{1}{4} \lambda_{5} V_{5}+\frac{1}{4} \lambda_{5}^{*} V_{5}^{*} .
\end{aligned}
$$

Written in terms of components the potential is

$$
\begin{aligned}
V= & -\mu^{2} \phi_{i \alpha} \phi_{i \alpha}^{*}+\frac{1}{2} \lambda_{1} \phi_{i \alpha} \phi_{i \alpha}^{*} \phi_{j \beta} \phi_{j \beta}^{*}+\frac{1}{2} \lambda_{2} \phi_{i \alpha} \phi_{i \beta}^{*} \phi_{j \beta} \phi_{j \alpha}^{*} \\
& +\frac{1}{2} \epsilon_{C} \lambda_{3} \phi_{i \alpha} C_{\alpha \beta} \phi_{j \beta} \phi_{j \gamma}^{*} C_{\gamma \delta} \phi_{i \delta}^{*} \\
& +\frac{1}{2} \epsilon_{D} \lambda_{4} \phi_{i \alpha} D_{i j} \phi_{j \beta} \phi_{k \beta}^{*} D_{k l} \phi_{l \alpha}^{*} \\
& +\epsilon_{D} \frac{1}{4} \lambda_{5} \phi_{j \alpha} C_{\alpha \beta} \phi_{k \beta} D_{k l} \phi_{l \gamma} C_{\gamma \delta} \phi_{i \delta} D_{i j} \\
& +\frac{1}{4} \lambda_{5}^{*} \phi_{j \alpha}^{*} C_{\alpha \beta} \phi_{k \beta}^{*} D_{k l} \phi_{l \gamma}^{*} C_{\gamma \delta} \phi_{i \delta}^{*} D_{i j}
\end{aligned}
$$

Note that the $\lambda_{1}$ term differs from all other four-point couplings because it has two disjoint index loops rather than one. The equations of motion break one loop into a string. Hence the $\lambda_{1}$ term contributes a factor with a closed index loop to the equations of motion. Therefore it contributes to the equations of motion through a trace over all nonzero elements of $\phi$. This explains why the $\lambda_{1}$ dependence is different from all other coupling dependencies.

In its most general form the potential has four real parameters, $\lambda_{1} \ldots \lambda_{4}$, and one complex one, $\lambda_{5}$. The most general form applies only to cases with both a $C$ and a $D$ matrix, namely, $O(N) \times O(M), U S p(2 N) \times U S p(2 M)$, and $O(N) \times U S p(2 M)$. However, in the first two of these cases we have to impose a reality condition. This implies that we can express all $\phi^{*}$ in terms of $\phi$. Then there are only two distinct quartic terms possible, namely, $V_{1}$, written as $(\phi \phi)^{2}$, and $V_{5}$. All other terms can be expressed in terms of products of four fields $\phi$, and since they have just one index loop they must all be related to $V_{5}$. Note that if a reality condition is imposed $V_{5}$ and $V_{5}^{*}$ are separately real, and $\lambda_{5}$ can be chosen to be real without loss of generality. Since $V_{2}, V_{3}$, and $V_{4}$ are all related to $V_{5}$, we may select one of them to our convenience. So we make a choice that is universally valid, and keep just the $\mu^{2}, \lambda_{1}$, and $\lambda_{2}$ term for $O(N) \times O(M)$ and $U S p(2 N) \times U S p(2 M)$. All terms in the potential are distinct only if the symmetry group is $O(N) \times U \operatorname{Sp}(2 M)$.

In Table I we list all potential terms that can occur. For future purposes we have chosen a certain order of the two group types in the last three cases.

\section{The vacuum energy}

Before getting into the details of solving the equations of motion, we derive here a useful general formula for the vacuum energy at the extremal points of potentials with only quadratic and quartic terms. Consider potentials of the form

$$
\begin{aligned}
V= & -\mu^{2} \phi_{x} \phi_{x}^{*}+\frac{1}{2} \lambda_{1}\left(\phi_{x} \phi_{x}^{*}\right)^{2}+\frac{1}{2} \sum_{i} \lambda_{i} T_{x y v w}^{i} \phi_{x} \phi_{y} \phi_{v}^{*} \phi_{w}^{*} \\
& +\frac{1}{4} \sum_{i} \rho_{i} P_{x y v w}^{i} \phi_{x} \phi_{y} \phi_{v} \phi_{w}+\frac{1}{4} \sum_{i} \rho_{i}^{*} P_{x y v w}^{i} \phi_{x}^{*} \phi_{y}^{*} \phi_{v}^{*} \phi_{w}^{*}
\end{aligned}
$$

The potential given above is of this form, with $x$ interpreted as the index pair $i \alpha$. Here we assume that $P^{i}$ and $T^{i}$ are real, as indeed they are in the case of interest. All indices $x, y, v$, $w$ are implicitly summed. Note that $T^{i}$ is symmetric in $x, y$ and $v, w$, and that $P^{i}$ is symmetric in all four indices $x y v w$. Furthermore we assume that $T^{i}$ satisfies $T_{x y v w}^{i}=T_{v w x y}^{i}$, so that all terms involving $T^{i}$ are real. The equations of motion, derived by differentiating with respect to $\phi_{z}^{*}$, are

$$
\begin{aligned}
\frac{\partial V}{\partial \phi_{z}^{*}}= & 0=-\mu^{2} \phi_{z}+\lambda_{1} \phi_{z}\left(\phi_{x} \phi_{x}^{*}\right)+\sum_{i} \lambda_{i} T_{x y v z}^{i} \phi_{x} \phi_{y} \phi_{v}^{*} \\
& +\sum_{i} \eta_{i}^{*} P_{x y v z}^{i} \phi_{x}^{*} \phi_{y}^{*} \phi_{v}^{*} .
\end{aligned}
$$


If we multiply this with $\phi_{z}^{*}$ and sum over $z$ we get

$$
\begin{aligned}
0= & -\mu^{2} \phi_{z} \phi_{z}^{*}+\lambda_{1}\left(\phi_{x} \phi_{x}^{*}\right)^{2}+\sum_{i} \lambda_{i} T_{x y v z}^{i} \phi_{x} \phi_{y} \phi_{v}^{*} \phi_{z}^{*} \\
& +\sum_{i} \eta_{i}^{*} P_{x y v z}^{i} \phi_{x}^{*} \phi_{y}^{*} \phi_{v}^{*} \phi_{z}^{*} .
\end{aligned}
$$

The first three terms on the left-hand side are real. Hence the equations of motion imply that

$$
\operatorname{Im} \sum_{i} \eta_{i}^{*} P_{x y v z}^{i} \phi_{x}^{*} \phi_{y}^{*} \phi_{v}^{*} \phi_{z}^{*}=0 .
$$

This implies that we may write

$$
\begin{aligned}
\sum_{i} \eta_{i}^{*} P_{x y v z}^{i} \phi_{x}^{*} \phi_{y}^{*} \phi_{v}^{*} \phi_{z}^{*}= & \frac{1}{2} \sum_{i} \eta_{i}^{*} P_{x y y z}^{i} \phi_{x}^{*} \phi_{y}^{*} \phi_{v}^{*} \phi_{z}^{*} \\
& +\frac{1}{2} \sum_{i} \eta_{i} P_{x y v z}^{i} \phi_{x} \phi_{y} \phi_{v} \phi_{z} .
\end{aligned}
$$

Substituting this into Eq. (5), we see that we can express all quartic potential contributions in terms of the mass terms, and that the value of $V$ at an extremum is always given by

$$
V_{\mathrm{EOM}}=-\frac{1}{2} \mu^{2} \phi_{x} \phi_{x}^{*},
$$

with implicit summation over $x$, as before. This conclusion does not hold if there are cubic terms in the potential.

\section{B. Symmetric bifundamentals}

In this section we consider the case of a bifundamental Higgs system with symmetry group $U(N) \times U(M)$, $O(N) \times O(M)$, and $U S p(N) \times U S p(M)$. We call these symmetric bifundamentals because the left and right group are of the same type. However, $N$ and $M$ may be different. Note that the two groups act independently. We make use of the fact that the field $\phi$ is complex, real, and quaternionic in these three cases, respectively. This allows a simultaneous derivation of the result in all three cases. As explained above, we can use the reality or quaternionic constraint to show that only the $\lambda_{1}$ and $\lambda_{2}$ terms in the potential contribute. The equations of motion are

$$
\mu^{2} \phi_{i \alpha}=\lambda_{1} \phi_{i \alpha}\left(\phi_{j \beta} \phi_{j \beta}^{*}\right)+\lambda_{2} \phi_{i \beta} \phi_{j \alpha} \phi_{j \beta}^{*} .
$$

This is obtained from the $\phi_{i \alpha}^{*}$ variation. We treat the field here as complex in all cases. In the quaternionic cases the fields belong to $2 \times 2$ blocks, but there is no need for indicating that. Strictly speaking the $\phi_{i \alpha}^{*}$ variation also acts on $\phi$ if there is a reality condition. But this just gives rise to an extra factor 2 in all contributing terms, and hence one gets exactly the same equation.

The easiest way to solve the problem is to make use of a singular value decomposition of $\phi$. This means that $\phi$ can be written as

$$
\phi=U R V,
$$

where $\quad U \in O(N), U(N)$ or $\quad U S p(N)$ and $\quad V \in$ $O(M), U(M)$ or $U S p(M)$ in the three cases, respectively. The matrix $R$ is diagonal, real, and non-negative. Note that negative diagonal elements can be made positive by a suitable one-sided $U(N)$ or $O(N)$ transformation. In the symplectic case $R$ must be diagonal in terms of quaternions, which means that it consists of $2 \times 2$ blocks $r \mathbb{1}$, with $r$ real and positive. Also in this case a negative sign can be flipped by a one-sided symplectic transformation [namely, $-\mathbb{1} \in S U(2) \subset U S p(N)]$. The singular value decomposition for quaternions has been derived in [6]. Note that a singular value decomposition works even if $\phi$ is not a square matrix.

Although singular value decomposition is less widely known than matrix diagonalization, they are used in the standard model of particle physics, namely, for the diagonalization of quark mass matrices. Since the latter are square matrices, one usually solves this problem by means of polar decompositions, which can be viewed as a special case of singular value decompositions. In [1] no use was made of singular value decompositions. Instead the Hermitian quantity $\phi \phi^{\dagger}$ was used, which can be diagonalized in the traditional way. But an extra step is needed to relate the diagonal form of $\phi \phi^{\dagger}$ to $\phi$ itself.

We can now arrive at the final answer in just a few steps. By a left and right gauge transformation one can remove $U$ and $V$, and hence one may replace $\phi$ by its eigenvalues, $\phi_{i \alpha}=r_{i} \delta_{i \alpha}, r_{i} \in \mathbb{R} ; r_{i} \geq 0$. Substituting this into the equations of motion we get

$$
\mu^{2} r_{i} \delta_{i \alpha}=\lambda_{1} r_{i} \delta_{i \alpha} \sum_{j} r_{j}^{2}+\lambda_{2} r_{i}^{3} \delta_{i \alpha}
$$

For those values of $i$ with $r_{i} \neq 0$ this implies

$$
\mu^{2}=\lambda_{1} \sum_{j} r_{j}^{2}+\lambda_{2} r_{i}^{2}
$$

which implies that all nonvanishing $r_{i}$ must be identical: $r_{i}=r$. Suppose there are $K$ nonvanishing ones. Then

$$
\mu^{2}=\lambda_{1} K r^{2}+\lambda_{2}^{2} r^{2}
$$

which means that (since $r \geq 0$ )

$$
r=\sqrt{\frac{\mu^{2}}{K \lambda_{1}+\lambda_{2}}} .
$$

The energy of these solutions is, from (7) or directly from the potential,

$$
E=-\frac{1}{2} \frac{\mu^{4} K}{K \lambda_{1}+\lambda_{2}}
$$




\section{Asymmetric bifundamentals}

\section{Why a new strategy is needed}

All methods used so far for the symmetric case, diagonalization of $\phi$ or $\phi \phi^{\dagger}$ or singular value decompositions, fail in all asymmetric cases, U-O, U-S, and O-S. Note first of all that $\phi$ is a general complex matrix in all these cases. No reality or quaternionic constraints can be imposed. Therefore $\phi$ is not diagonalizable by means of the available gauge symmetries. Quantities like $\phi \phi^{\dagger}$ are diagonalizable in some cases, but the potential has additional terms, and cannot be expressed in terms of just one of the matrices $\phi \phi^{\dagger}, \phi^{\dagger} \phi, \phi \phi^{T}$, or $\phi^{T} \phi$. If there is more than one, they would have to be diagonalized simultaneously. Furthermore for the diagonalization of some of these quantities unitary transformations are required, while only orthogonal or symplectic ones are available.

None of the known theorems concerning singular value decompositions apply to U-O, U-S, or O-S. In other words, one may of course diagonalize any complex matrix $\phi$ by means of unitary matrices, but it is not possible to gauge these transformation matrices away. One may speculate about the possibility that there exists some generalization of singular vector decompositions that allows us to write $\phi$ in a simpler form. While some version of that statement may be correct, some concrete guesses can be ruled out by counting parameters. For example, compare $U(N) \times U(N)$ and $O(N) \times U S p(N)$ (we focus on square matrices here). In both cases the field $\phi$ is complex and has $2 N^{2}$ real parameters, precisely the same as the number of parameters of $U(N) \times U(N)$. The difference between these numbers determines the minimal number of parameters that remains after gauge fixing; the actual number can be larger because of degeneracies in the action of the gauge symmetries. Indeed, in this case the minimal number of parameters is 0 , but the actual number is $N$ because common phases in the left and right unitary groups have the same effect. For $O(N) \times O(N)$ the minimal number is $N$, which is also the actual number, and for $U S p(N) \times U S p(N)$ with a quaternionic condition the minimal number is $-N$, and the actual number $\frac{1}{2} N$.

But for $O(N) \times U S p(N)$ the minimal number of remaining parameters is

$$
2 N^{2}-\frac{1}{2} N(N-1)-\frac{1}{2} N(N+1)=N^{2} .
$$

This immediately ruins any chance for a diagonal form, and even for some more general block-diagonal form. If the putative final result is a block-diagonal matrix with $(N / p)$ complex $p \times p$ blocks, the total number of parameters is $2 N p$. Only for $p=N / 2$ one can just saturate the bound, but then the matrix just decomposes into two general complex $N / 2 \times N / 2$ matrices. Even if this were possible, it does not look like a useful result. So we need an entirely different strategy.

\section{Equations of motion}

We have just seen that the procedure of first bringing the fields in the simplest form using gauge rotations, and only then applying the equations of motion, fails. Our strategy is therefore to intertwine these two tools in several steps: simplify by gauge rotations, apply the equations of motion, simplify further by another gauge rotation, and then use the equations of motion once more.

We consider the potential with all five quartic terms, but one has to keep in mind that in some cases some of the parameters may vanish. The equations of motion, obtained by varying the potential with respect to $\phi_{i \alpha}^{*}$, are

$$
\begin{aligned}
\mu^{2} \phi_{i \alpha}= & \lambda_{1} \phi_{i \alpha}\left(\phi_{j \beta} \phi_{j \beta}^{*}\right)+\lambda_{2} \phi_{i \beta} \phi_{j \alpha} \phi_{j \beta}^{*} \\
& +\epsilon_{C} \lambda_{3} \phi_{i \delta} C_{\delta \beta} \phi_{j \beta} \phi_{j \gamma}^{*} C_{\gamma \alpha} \\
& +\epsilon_{D} \lambda_{4} \phi_{m \alpha} D_{m j} \phi_{j \beta} \phi_{k \beta}^{*} D_{k i} \\
& +\lambda_{5}^{*} C_{\alpha \beta} \phi_{j \beta}^{*} D_{j k} \phi_{k \gamma}^{*} C_{\gamma \delta} \phi_{m \delta}^{*} D_{m i}
\end{aligned}
$$

We may distinguish two kinds of equations of motion: those for $\phi_{i \alpha}=0$ and those for $\phi_{i \alpha} \neq 0$. In equations of the former kind, both the $\mu^{2}$ term and the $\lambda_{1}$ term drop out, and one is left with the last four terms. We call two kinds of equations "homogeneous" and "inhomogeneous" respectively because in the first kind all terms are cubic. This is a slight abuse of the terminology commonly used for linear equations.

We do not consider solutions that only work for special values of $\lambda_{i}$ or special relations among the parameters $\lambda_{i}$. Such relations are not renormalization group invariant unless the potential has some additional symmetry. This requirement rules out cancellations among the four cubic terms with coefficients $\lambda_{2} \ldots \lambda_{5}$, the four terms of a homogeneous equation. Each must vanish separately, and hence for each homogeneous equation we get up to four equations, one for each coupling constant.

Most of the information in the inhomogeneous equations can be dealt with in the same way: one can derive a second class of homogeneous equations from them. Consider two nonvanishing elements $\phi_{i \alpha}$ and $\phi_{k \gamma}$. From two inhomogeneous equations we can obtain a homogeneous one by multiplying the equations for $\phi_{i \alpha}$ by $\phi_{k \gamma}$ and vice versa, and subtracting the two. Then the $\mu^{2}$ and $\lambda_{1}$ terms drop out. The resulting difference equation must be satisfied for generic values of $\lambda_{p}$, and hence it splits into four separate equations, labeled by the index $p$ of the coupling constants $\lambda_{p}$.

This argument cannot be applied to the inhomogeneous equations because they have parameters of different dimensions. If all terms in the equation have the same tensor structure they can be made to cancel by changing the overall scale of the field. Hence such cancellations do not 
depend on special, fixed relations between $\mu^{2}$ and the coupling constants.

In order to make the metric $C$ and $D$ explicit we assume that the symplectic groups act only on the column basis (labeled by $\alpha, \beta, \ldots$ ), and orthogonal ones only on the row basis $(i, j, \ldots)$. This allows us to consider the four cases $U(N) \times U(M), \quad O(N) \times U(M), \quad U(N) \times U S p(M), \quad$ and $O(N) \times U S p(M)$. Although $U(N) \times U(M)$ has already been solved, we include it for illustrative purposes. Now we can replace $D$ by a Kronecker $\delta$ and $C$ by an antisymmetric matrix $\Omega$. Since $\Omega$ pairs indices, we absorb it in most cases in the fields by defining

$$
\phi_{i \tilde{\alpha}}=\Omega_{\alpha \beta} \phi_{i \beta}
$$

so that the indices $\alpha$ and $\tilde{\alpha}$ form a symplectic pair. The remaining two types, $O(N) \times O(M)$ and $U S p(N) \times$ $U S p(M)$, cannot be treated in this way. Nevertheless these cases can also be solved by the following method, by applying it to real numbers or quaternions. But because these cases have already been solved, we do not discuss this.

For the special case where $G(N)$ is either $U(N)$ or $O(N)$ and $H(M)$ is either $U(M)$ or $U S p(M)$ the equations of motion for $\phi_{i \alpha}$ read

$$
\begin{aligned}
\mu^{2} \phi_{i \alpha}= & \lambda_{1} \phi_{i \alpha}\left(\phi_{j \beta} \phi_{j \beta}^{*}\right)+\lambda_{2} \phi_{i \beta} \phi_{j \alpha} \phi_{j \beta}^{*}+\lambda_{3} \phi_{i \beta} \phi_{j \tilde{\beta}} \phi_{j \tilde{\alpha}}^{*} \\
& +\lambda_{4} \phi_{j \alpha} \phi_{j \beta} \phi_{i \beta}^{*}+\lambda_{5}^{*} \phi_{j \tilde{\alpha}}^{*} \phi_{j \beta}^{*} \phi_{i \tilde{\beta}}^{*} .
\end{aligned}
$$

The homogeneous equations are simply that the four terms with coefficients $\lambda_{p}, p=2 \ldots 5$ must vanish if $\phi_{i \alpha}=0$ (note that the $\lambda_{1}$ term also vanishes in that case). The second class of homogeneous equations mentioned above, the weighted difference of two inhomogeneous equations for nonzero fields $\phi_{i \alpha}$ and $\phi_{k \gamma}$, yields

$$
\begin{aligned}
G \times H: \phi_{i \beta} \phi_{j \alpha} \phi_{j \beta}^{*} \phi_{k \gamma} & =\phi_{k \beta} \phi_{j \gamma} \phi_{j \beta}^{*} \phi_{i \alpha}, \\
G \times S: \phi_{i \beta} \phi_{j \tilde{\beta}} \phi_{j \tilde{\alpha}}^{*} \phi_{k \gamma} & =\phi_{k \beta} \phi_{j \tilde{\beta}} \phi_{j \tilde{\gamma}}^{*} \phi_{i \alpha}, \\
O \times H: \phi_{j \alpha} \phi_{j \beta} \phi_{i \beta}^{*} \phi_{k \gamma} & =\phi_{j \gamma} \phi_{j \beta} \phi_{k \beta}^{*} \phi_{i \alpha}, \\
O \times S: \phi_{j \tilde{\alpha}}^{*} \phi_{j \beta}^{*} \phi_{i \tilde{\beta}}^{*} \phi_{k \gamma} & =\phi_{j \tilde{\gamma}}^{*} \phi_{j \beta}^{*} \phi_{k \tilde{\beta}}^{*} \phi_{i \alpha}
\end{aligned}
$$

Not all these equations are available in all cases; this depends on $p$ as indicated in Table I. We have indicated this here by $G=U$ or $O, H=U$ or $U S p$, and $S$ is used as shorthand for $U S p$. These relations are implicitly summed over $\beta$ and $j$.

\section{Equations for pivot elements}

It turns out to be sufficient to study these equations for special cases where the row and column of a certain element $\phi_{i j} \neq 0$ consists mostly of 0 's. Consider first the special case where a row $i$ contains only one element, labeled by column index $\delta(i)$,

$$
\phi_{i \delta(i)} \neq 0 ; \quad \phi_{i \alpha}=0 \quad \text { for all } \alpha \neq \delta(i) .
$$

We call such an element $\phi_{i \delta(i)}$ a pivot element.

There is always at least one such element, because one can always bring one row into that form using either unitary or symplectic column transformations (note that in our setup orthogonal transformations do not act on the column indices). This works somewhat differently in the unitary and symplectic case, and the details are explained in the Appendix. Obviously one can do this for just one row or column at a time. We may also use the row and column symmetries to set $i=\delta(i)=1$, but we leave the notation general for now.

The existence of such a row implies that there are $N-1$ homogeneous equations due to $\phi_{i \alpha}=0$. Furthermore, since there is only one nonzero element, the sum over $\beta$ collapses to a single term. Each of the four homogeneous equations can be divided by $\phi_{i \delta(i)} \neq 0$. Then we get

$$
\begin{aligned}
& G \times H: \phi_{j \alpha} \phi_{j \delta(i)}^{*}=0, \\
& G \times S: \phi_{j \tilde{\delta}(i)} \phi_{j \tilde{\alpha}}^{*}=0 \\
& O \times H: \phi_{j \alpha} \phi_{j \delta(i)}=0, \\
& O \times S: \phi_{j \tilde{\alpha}} \phi_{j \tilde{\delta}(i)}=0 .
\end{aligned}
$$

This implies that in general, every column must be complex orthogonal $\left(\vec{v} \cdot \vec{w}^{*}=0\right)$ to the column with the pivot element. In the symplectic case, every column must in

TABLE I. Potential terms.

\begin{tabular}{lcccc}
\hline \hline Groups & $D$ & $C$ & Reality condition & Coupling constants \\
\hline$U(N) \times U(M)$ & None & None & None & $\lambda_{1}, \lambda_{2}$ \\
$O(N) \times O(M)$ & $\delta$ & $\delta$ & $\phi=\phi^{*}$ & $\lambda_{1}, \lambda_{2}$ \\
$U S p(2 N) \times U S p(2 M)$ & $\Omega$ & $\Omega$ & $\phi^{*}=\Omega^{T} \phi \Omega$ & $\lambda_{1}, \lambda_{2}$ \\
$O(N) \times U(M)$ & $\delta$ & None & None & $\lambda_{1}, \lambda_{2}, \lambda_{4}$ \\
$U(N) \times U S p(2 M)$ & None & $\Omega$ & None & $\lambda_{1}, \lambda_{2}, \lambda_{3}$ \\
$O(N) \times U S p(2 M)$ & $\delta$ & $\Omega$ & None & $\lambda_{1}, \lambda_{2}, \lambda_{3}, \lambda_{4}, \lambda_{5}$ \\
\hline \hline
\end{tabular}


addition be complex orthogonal to the column $\tilde{\delta}$ paired with the pivot element column. Furthermore the column $\tilde{\delta}$ must be complex orthogonal to column $\delta$. In the $O(N)$ case the same statements must hold for both complex and real orthogonality $(\vec{v} \cdot \vec{w}=0)$.

Now consider inhomogeneous difference equations for pivot elements. This requires a second row $k$ with a pivot element in column $\delta(k)$. Having two such rows cannot be arranged purely by gauge rotations, but we need this later in an intermediate step. Assuming two rows with pivot elements $\phi_{i \delta(i)}$ and $\phi_{k \delta(k)}$ we get in the four cases respectively (with implicit sums over $j$ )

$$
\begin{aligned}
& G \times H: \phi_{j \delta(i)} \phi_{j \delta(i)}^{*}=\phi_{j \delta(k)} \phi_{j \delta(k),}^{*}, \\
& G \times S: \phi_{j \tilde{\delta}(i)} \phi_{j \tilde{\delta}(i)}^{*}=\phi_{j \tilde{\delta}(k)} \phi_{j \tilde{\delta}(k),}^{*},
\end{aligned}
$$

$O \times H: \phi_{j \delta(i)} \phi_{j \delta(i)} \phi_{i \delta(i)}^{*} \phi_{k \delta(k)}=\phi_{j \delta(k)} \phi_{j \delta(k)} \phi_{k \delta(k)}^{*} \phi_{i \delta(i),}$

$O \times H: \phi_{j \tilde{\delta}(i)}^{*} \phi_{j \tilde{\delta}(i)}^{*} \phi_{i \delta(i)}^{*} \phi_{k \delta(k)}=\phi_{j \tilde{\delta}(k)}^{*} \phi_{j \tilde{\delta}(k)}^{*} \phi_{k \delta(k)}^{*} \phi_{i \delta(i)}$

In the first two equations we have divided by the nonvanishing element $\phi_{i \delta(i)}$ and $\phi_{k \delta(k)}$. In the last two this is not possible, because these factors appear as conjugates on the left- and right-hand side. The first two equations imply that any two columns containing at least one pivot element must have the same norm. Furthermore, in the symplectic case, their symplectic conjugate columns $\tilde{\delta}(i)$ and $\tilde{\delta}(k)$ must have the same norms as well. Note that this is true even if the columns $\tilde{\delta}(i)$ and $\tilde{\delta}(k)$ do not contain a pivot element themselves. If one of the symplectic conjugate columns $\tilde{\delta}(i)$ or $\tilde{\delta}(k)$ contains a pivot element, then all four columns $\delta(i), \tilde{\delta}(k), \tilde{\delta}(i)$, and $\tilde{\delta}(k)$ must have the same norm.

A special case of interest is that of two or more pivot elements appearing in the same column. Then the first two equations are trivially satisfied, but now the third and fourth equations add new information. Suppose we have at least two row labels $i$ and $k$ with $\delta(i)=\delta(k) \equiv \delta$. Since all other elements on these rows vanish we can make unitary or orthogonal rotations on these rows, which allow us to bring the $\delta$-column vector $\left(\phi_{i \delta}, \phi_{k \delta}, \ldots\right)$ into a special form. The dots indicate any additional pivot elements on column $\delta$. If $G=U$ we can rotate the column so that only $\phi_{i \delta} \neq 0$. This case is of no further interest, since now we have only a single pivot element. If $G=O$ we have orthogonal transformations acting on complex vectors. Then we can rotate the $\delta$ column to the form $\phi_{i \delta}=x$ and $\phi_{k \delta}=r$. If $x=x^{*}$ we can rotate $r$ to 0 , so that we have only a single pivot element, and nothing new can be learned. So assume that $x \neq x^{*}$ and $r \neq 0$. Then we find from the last two equations, after dividing by $\left(x-x^{*}\right) r$,

$$
\begin{aligned}
& \sum_{j} \phi_{j \delta} \phi_{j \delta}=0 \text { for } O(N) \times U(N) \text { or } O(N) \times U S p(N), \\
& \sum_{j} \phi_{j \tilde{\delta}} \phi_{j \tilde{\delta}}=0 \text { for } O(N) \times U S p(N) .
\end{aligned}
$$

These equations are especially useful if a column contains exactly two pivot elements, because then it implies that these elements must differ by a factor $i$.

\section{The inhomogeneous equation}

Now we turn to the inhomogeneous equations. They have the form (9). For a given solution, we may write all nonvanishing elements as

$$
\phi_{i \alpha}=r \mu \chi_{i \alpha} .
$$

The functions $\chi_{i \alpha}$ are dimensionless, and can be given a standard normalization by setting one of them to 1 . To define $r$ we have to find some canonical definition of one special nonzero element $\phi_{i \alpha}$. This can be done as follows. First we work out the row norms $n_{i}=\sum_{\alpha} \phi_{i \alpha} \phi_{i \alpha}^{*}$. These are invariant under all column basis transformations $H(M)$, because all column transformations are either $U(M)$ or a subgroup of $U(M)$. We consider arbitrary $G(N)$ transformations of the rows $n_{i}$ to maximize the largest norm in this set. The row with the largest possible norm is then $G(N)$ transformed to row 1 . This is possible for any choice of $G(N)$. Using an $H(M)$ transformation we now rotate row 1 so that only $\phi_{11}$ is nonzero, real, and positive, as explained above. Now we define $r$ in such a way that $\chi_{11}=1$. Then we have obtained a basis so that

$$
\chi_{11}=1 ; \quad \chi_{1 \alpha}=0 \quad \text { for all } \alpha \geq 2 .
$$

This procedure implies that $\left|\chi_{i \alpha}\right| \leq 1$ for all $i$ and $\alpha$. In terms of this parametrization the inhomogeneous equation now becomes

$1=r^{2}\left[\lambda_{1} P+\lambda_{2} \chi_{j 1} \chi_{j 1}^{*}+\lambda_{3} \chi_{j 2} \chi_{j 2}^{*}+\lambda_{4} \chi_{j 1} \chi_{j 1}+\lambda_{5}^{*} \chi_{j 2}^{*} \chi_{j 2}^{*}\right]$,

where $P=\sum \chi_{i \alpha} \chi_{i \alpha}^{*}$. Note that by construction $\phi_{11}$ is real, and by definition $\chi_{11}=1$. Furthermore $\mu$ is real, so $r$ must be real as well. But this is not manifest in this equation: the last two terms are not manifestly real. However, clearly their sum must be real, and since we do not allow solutions that require special relations between parameter values, this must imply that they are separately real. We define

$$
\begin{aligned}
& \rho_{2}=\chi_{j 1} \chi_{j 1}^{*}, \\
& \rho_{3}=\chi_{j 2} \chi_{j 2}^{*}, \\
& \rho_{4}=\chi_{j 1} \chi_{j 1}, \\
& \rho_{5}=\chi_{j 2}^{*} \chi_{j 2}^{*} .
\end{aligned}
$$


Then we get

$$
r=\sqrt{\frac{1}{P \lambda_{1}+Q}}
$$

where

$$
\begin{aligned}
& P=\sum_{i=2} \chi_{i \alpha} \chi_{i \alpha}^{*}, \\
& Q=\sum_{i=2}^{4} \lambda_{i} \rho_{i}+\lambda_{5}^{*} \rho_{5} .
\end{aligned}
$$

In the special basis we have chosen, $\chi_{11}=1$, and $\chi_{1 \beta}=0$ for $\beta>1$. This implies that we can write out the sum over $\beta$,

$$
\rho_{2}=\chi_{11} \sum_{j=1}^{N} \chi_{j 1} \chi_{j 1}^{*}=1+\sum_{j=2}^{N} \chi_{j 1} \chi_{j 1}^{*} \geq 1 .
$$

Using row transformations acting on the last $N-1$ rows we can always bring the first column in a simpler form. If $G(N)=U(N)$, we can rotate all elements except $\chi_{21}$ to zero values. If $G(N)=O(N)$ we can bring $\chi_{21}$ to a general complex value, and $\chi_{31}$ to a positive real value. Since row 1 has norm 1, and since the norms were maximized using $G(N)$, we have $\left|\chi_{21}\right| \leq 1$ and $\left|\chi_{31}\right| \leq 1$. Hence $\rho_{2} \leq 3$. In practice the maximum value attained by $\rho_{2}$ turns out to be 2 .

The values of $\rho_{p}$ can be directly related to the potential. Consider first the inhomogeneous equation for any other nonvanishing field $\phi_{k \gamma}$,

$$
\begin{aligned}
\chi_{k \gamma}= & r^{2}\left[\lambda_{1} \chi_{k \gamma}\left(\chi_{j \beta} \chi_{j \beta}^{*}\right)+\lambda_{2} \chi_{k \beta} \chi_{j \gamma} \chi_{j \beta}^{*}+\lambda_{3} \chi_{k \beta} \chi_{j \tilde{\beta}} \chi_{j \tilde{\gamma}}^{*}\right. \\
& \left.+\lambda_{4} \chi_{j \gamma} \chi_{j \beta} \chi_{k \beta}^{*}+\lambda_{5}^{*} \chi_{j \tilde{\gamma}}^{*} \chi_{j \beta}^{*} \chi_{k \tilde{\beta}}^{*}\right] .
\end{aligned}
$$

By subtracting (18) times $\chi_{k \gamma}$ and using the principle that cancellations depending on special relations among the $\lambda$ 's are not acceptable, we get

$$
\begin{aligned}
& \sum_{j, \beta} \chi_{k \beta} \chi_{j \gamma} \chi_{j \beta}^{*}=\rho_{2} \chi_{k \gamma}, \\
& \sum_{j, \beta} \chi_{k \beta} \chi_{j \tilde{\beta}} \chi_{j \tilde{\gamma}}^{*}=\rho_{3} \chi_{k \gamma}, \\
& \sum_{j, \beta} \chi_{j \gamma} \chi_{j \beta} \chi_{k \beta}^{*}=\rho_{4} \chi_{k \gamma}, \\
& \sum_{j, \beta} \chi_{j \tilde{\gamma}}^{*} \chi_{j \beta}^{*} \chi_{k \tilde{\beta}}^{*}=\rho_{5} \chi_{k \gamma} .
\end{aligned}
$$

These equations are obtained here for $\chi_{k \gamma} \neq 0$, but it also holds for $\chi_{k \gamma}=0$, because then they are just the homogeneous equations. Now we multiply both sides of these relations with $\chi_{k \gamma}^{*}$ and sum over $k$ and $\gamma$. Then we get, in terms of the potentials $V_{p}$ defined in (3),

$$
\begin{aligned}
& \rho_{p}=\frac{V_{p}}{\mu^{4} r^{4} P} \quad \text { for } p=2,3,4, \\
& \rho_{5}=\frac{V_{5}^{*}}{\mu^{4} r^{4} P} .
\end{aligned}
$$

These expressions show, in particular, that $\rho_{p}$ is gauge invariant, which was not manifest in the construction we gave above. Note that $\rho_{2}$ and $\rho_{3}$ are manifestly real because of their definition (19). Furthermore, $\rho_{4}$ is proportional to $V_{4}$, which is manifestly real, but this proportionality holds only for solutions of the equation of motion. The coupling constants $\lambda_{2}, \lambda_{3}$, and $\lambda_{4}$ are real, and since $Q$ must be real this implies that $\rho_{5} \lambda_{5}^{*}$ must be real, although in general neither $\rho_{5}$ nor $\lambda_{5}$ are real themselves. This implies

$$
\lambda_{5}^{*} \rho_{5}= \pm\left|\lambda_{5}\right|\left|\rho_{5}\right| .
$$

Hence for solutions to the equations of motion $\lambda_{5} V_{5}$ is real. Therefore

$$
\lambda_{5}^{*} V_{5}^{*}=\frac{1}{2}\left(\lambda_{5} V_{5}+\lambda_{5}^{*} V_{5}^{*}\right) .
$$

Using this result and (3) we can express the entire quartic contribution to the potential in terms of the $\rho$ parameters and $P$,

$$
\begin{aligned}
V & =-\mu^{2} \phi_{i \alpha} \phi_{i \alpha}^{*}+\frac{1}{2} \mu^{4} r^{4} P\left(\lambda_{1} P+\lambda_{2} \rho_{2}+\lambda_{3} \rho_{3}+\lambda_{4} \rho_{4}+\lambda_{5}^{*} \rho_{5}\right) \\
& =-\mu^{4} r^{2} P+\frac{1}{2} \mu^{4} r^{4} P\left(\lambda_{1} P+Q\right) \\
& =-\frac{1}{2} \mu^{4} r^{2} P
\end{aligned}
$$

where in the last step we used (20). This result is consistent with the general formula (7).

\section{Disjoint solutions}

If one considers Higgs potentials for $G(N) \times H(M)$ group combinations, solutions must exist already for the smallest allowed values of $N$ and $M$ : if $\mu^{2}<0$, then $\phi=0$ is not a minimum, and hence if the potential is bounded there must exist a nontrivial minimum. These solutions remain valid if one enlarges $N$ and $M$ and chooses all additional elements of $\phi_{i \alpha}$ to be 0 .

Now consider two such solutions to the equations of motion, $\phi^{A}$ and $\phi^{B}$. One may attempt to combine two or more solutions, by choosing disjoint block submatrices of $\phi_{i \alpha}$ and embedding a known solution in it. Here by disjoint we mean first of all that no rows or columns exist with nonzero elements of both $\phi^{A}$ and $\phi^{B}$. But we need a slightly more general notion of nonoverlapping, namely, one that includes the effect of the matrices $D_{i j}$ or $C_{\alpha \beta}$. In practice these matrices are either diagonal or block diagonal in terms of $2 \times 2$ blocks, as happens for symplectic groups. In 
that case we combine rows or columns into pairs linked by $C$ or $D$, and we extend the notion of disjoint to pairs of rows or columns.

The homogeneous equations are automatically satisfied for combinations of solutions in disjoint sub-blocks. This is because nonzero elements of $\phi_{i \alpha}$ only connect indices belonging to the corresponding solution.

But this is not true for the inhomogeneous equations because of the $\lambda_{1}$ term. This includes a sum over all $\phi_{j \beta} \phi_{j \beta}^{*}$, which changes if solutions are added.

Consider two distinct solutions $\chi$ and $\xi$, each satisfying

$$
\begin{aligned}
& \chi_{i \alpha}=\lambda_{1} \chi_{i \alpha} r_{1}^{2} P_{1}+\lambda_{2} r_{1}^{2} \chi_{i \beta} \chi_{j \alpha} \chi_{j \beta}^{*}+\cdots \\
& \xi_{p \mu}=\lambda_{1} \xi_{p \mu} r_{2}^{2} P_{2}+\lambda_{2} r_{2}^{2} \xi_{p \nu} \xi_{q \mu} \xi_{q \nu}^{*}+\cdots
\end{aligned}
$$

where the indices $(i, \alpha)$ and $(p, \mu)$ are disjoint in the way explained above. Here we use the dimensionless unit introduced above; the two solutions are

$$
\begin{array}{ll}
\phi_{i \alpha}^{1}=r_{1} \chi_{i \alpha} ; \quad & r_{1}=\sqrt{\frac{1}{P_{1} \lambda_{1}+Q_{1}}}, \\
\phi_{i \alpha}^{2}=r_{2} \xi_{i \alpha} ; & r_{2}=\sqrt{\frac{1}{P_{2} \lambda_{1}+Q_{2}}},
\end{array}
$$

with

$$
P_{1}=\sum_{i, \alpha} \chi_{i \alpha} \chi_{i \alpha}^{*}, \quad P_{2}=\sum_{i, \alpha} \chi_{p \mu} \chi_{p \mu}^{*}
$$

This combined solution satisfied the homogeneous equations, but the inhomogeneous ones become

$$
\begin{aligned}
& \chi_{i \alpha}=\lambda_{1} \chi_{i \alpha}\left(r_{1}^{2} P_{1}+r_{2}^{2} P_{2}\right)+\lambda_{2} r_{1}^{2} \chi_{i \beta} \chi_{j \alpha} \chi_{j \beta}^{*}+\cdots, \\
& \xi_{p \mu}=\lambda_{1} \xi_{p \mu}\left(r_{1}^{2} P_{1}+r_{2}^{2} P_{2}\right)+\lambda_{2} r_{2}^{2} \xi_{p \nu} \xi_{q \mu} \xi_{q \nu}^{*}+\cdots
\end{aligned}
$$

Since the homogeneous equations are invariant under a simultaneous rescaling of all fields, one may hope that we can solve these equations by a rescaling

$$
r_{1} \rightarrow S_{1} r_{1} ; \quad r_{2} \rightarrow S_{2} r_{2}
$$

Now the inhomogeneous equations are

$$
\begin{aligned}
& \chi_{i \alpha}=\lambda_{1} \chi_{i \alpha}\left(S_{1}^{2} r_{1}^{2} P_{1}+S_{2}^{2} r_{2}^{2} P_{2}\right)+S_{1}^{2} r_{1}^{2} \lambda_{2} \chi_{i \beta} \chi_{j \alpha} \chi_{j \beta}^{*}+\cdots, \\
& \xi_{p \mu}=\lambda_{1} \xi_{p \mu}\left(S_{1}^{2} r_{1}^{2} P_{1}+S_{2}^{2} r_{2}^{2} P_{2}\right)+S_{2}^{2} r_{2}^{2} \lambda_{2} \xi_{p \nu} \xi_{q \mu} \xi_{q \nu}^{*}+\cdots
\end{aligned}
$$

Now we subtract $S_{1}^{2}$ times the single solution equation of motion (23) from the first, and analogously for the second. Then the $\lambda_{p}$ terms for $p \geq 2$ cancel, and we can divide by $\chi_{i \alpha}$, respectively $\xi_{p \mu}$. Then we solve for $S_{1}^{2}$ and $S_{2}^{2}$ and we get

$$
S_{1}^{2}=\frac{1-\lambda_{1} r_{2}^{2} P_{2}}{1-\lambda_{1}^{2} r_{1}^{2} r_{2}^{2} P_{1} P_{2}} \quad S_{2}^{2}=\frac{1-\lambda_{1} r_{1}^{2} P_{1}}{1-\lambda_{1}^{2} r_{1}^{2} r_{2}^{2} P_{1} P_{2}}
$$

Note that if we combine two identical solutions $\left(r_{1}=r_{2}\right.$, $\left.P_{1}=P_{2}\right)$ we find

$$
S_{1}^{2}=S_{2}^{2}=\frac{1}{1+\lambda_{1} r^{2} P}
$$

so that the $r$ parameter $r_{\text {comb }}$ of the combined solution is

$$
S_{1}^{2} r_{2}=S_{2}^{2} r_{2}^{2}=r_{\mathrm{comb}}^{2}=\frac{1}{2 P \lambda_{1}+Q} .
$$

It is easy to show that this process can be continued, and that for a combination of $K$ identical solutions the result is

$$
r^{2}=\frac{1}{K P \lambda_{1}+Q} .
$$

This can be verified by working out the combination of two multisolutions, one built out of $K_{1}$ and one build out of $K_{2}$ basic solutions. We can do that in general, for one $K_{1}$-fold solution with parameters $P_{1}$ and $Q_{1}$, and one $K_{2}$-fold solution with parameters $P_{2}$ and $Q_{2}$.

We find

$$
S_{1}^{2} r_{1}^{2}=\frac{Q_{2}}{\lambda_{1}\left(K_{2} P_{2} Q_{1}+K_{1} P_{1} Q_{2}\right)+Q_{1} Q_{2}}
$$

with an analogous formula for $S_{2}^{2} r_{2}^{2}$, with "1" and "2" interchanged. We see that if $P_{1}=P_{2}$ and $Q_{1}=Q_{2}$, then indeed we get the expected result for a $\left(K_{1}+K_{2}\right)$-fold solution. Furthermore we see then that $S_{1}^{2} r_{1}^{2}=S_{2}^{2} r_{2}^{2}$ if and only if $Q_{1}=Q_{2}$. For generic $\lambda_{i}$ we can have equality of $Q_{1}$ and $Q_{2}$ if and only if $\rho_{i}^{(1)}=\rho_{i}^{(2)}$ (the upper index labels the solution).

Note that even for distinct solutions, there is always a solution for the scale factors $S_{1}$ and $S_{2}$. But now we can consider the rescaled equations (24) for the special normalizing fields we have chosen to define $\rho_{i}$. These are the field $\chi_{11}=1$ chosen earlier, and the analogous choice for $\xi$. We can divide the equations by these fields and subtract them. Then we find

$$
S_{1}^{2} r_{1}^{2} \rho_{i}^{(1)}=S_{2}^{2} r_{2}^{2} \rho_{i}^{(2)}
$$

Using Eq. (25) we can write this as

$$
Q_{2} \rho_{i}^{(1)}=Q_{1} \rho_{i}^{(2)} .
$$

Note that if we contract this with $\lambda_{i}$, summing over $i=2 \ldots .5$, this is automatically satisfied. Without summation, these equations imply that two solutions can only be combined if their values of $\rho_{p}$ for different $p$ have the same ratios. 


$$
\frac{\rho_{i}^{(1)}}{\rho_{i}^{(2)}}=\frac{Q_{1}}{Q_{2}} .
$$

Hence, in particular, they must be simultaneously 0 . Furthermore we can divide (26) on both sides by the $i=$ 2 equation, which is always nontrivial. This implies that for two solutions to be combined, one must have

$$
\rho_{p}^{(2)}=\left[\frac{\rho_{2}^{(1)}}{\rho_{2}^{(2)}}\right] \rho_{p}^{(1)} .
$$

Although this still allows a common scaling, as we will see there are no two cases where the $\rho_{p}$ parameters differ only by a common scale.

\section{The main argument}

Now we combine all the foregoing results. As already discussed in Sec. II C 4, we can always rotate the first row to the form

$$
\left(\phi_{11}, 0, \ldots, 0\right)
$$

Then the homogeneous equation for $G \times H$ implies that all columns are orthogonal to column 1 . Now row 1 is fixed,

$$
\begin{array}{lll}
\mathbf{a}: \phi_{j 1}=0 & \text { for } j \geq 2, & \\
\mathbf{b}: \phi_{j 1}=0 & \text { for } j \geq 3 ; & \phi_{21} \neq 0 ; \\
\mathbf{c}: \phi_{j 1}=0 & \text { for } j \geq 4 ; & \phi_{21} \neq 0 ;
\end{array}
$$

Note that the last condition is that $\phi_{21}$ and $\phi_{31}$ have a different phase. If that were not the case we could rotate $\phi_{31}$ into $\phi_{21}$ and then case $\mathrm{c}$ turns into case $\mathrm{b}$. In case a we have a disjoint $1 \times 1$ block matrix if $H(M)=U(M)$ and the discussion is as before. In case $b$ the general orthogonality equation (10) implies that $\phi_{2 \alpha}=0$ for $\alpha \geq 2$. Then either $\phi_{21}$ is real, and it can be rotated into $\phi_{11}$, or it is not real, and the arguments at the end of Sec. II C 3 show that $\phi_{21}=i \phi_{11}$. In case c we use both (10) and (12) plus the fact that $\phi_{21}$ and $\phi_{31}$ have a different phase to show that $\phi_{2 \alpha}=\phi_{3 \alpha}=0$ for $\alpha \geq 2$. Now we have an $O(3)$ gauge symmetry in the first three rows at our disposal to reduce case $\mathrm{c}$ to case $\mathrm{b}$. We may now normalize $\phi_{11}$ to 1 by defining the parameter $r$ appropriately. We find then that we have two possible disjoint solutions. One works for both $U(N)$ and $O(N)$, and is characterized by an upperleft $1 \times 1$ block,

$$
A:(1)
$$

The other holds only for $O(N)$ and is characterized by an upper-left $2 \times 1$ block, but we have $U(N-1)$ or $O(N-1)$ rotations at our disposal to clean up the last $N-1$ entries of column 1 .

Consider first unitary column transformations. Then we can set $\phi_{j 1}=0$ for $j \geq 3$, and make $\phi_{21}$ real. Since all remaining columns must be orthogonal to column 1 , this implies that $\phi_{2 \alpha}=0$ for $\alpha \geq 2$. Now the first two rows of the matrix have the form

$$
\left(\begin{array}{llll}
\phi_{11} & 0 & \ldots & 0 \\
\phi_{21} & 0 & \ldots & 0
\end{array}\right) .
$$

If $\phi_{21}=0$, we have obtained a matrix where $\phi_{11}$ is the only nonzero element in the first row and column. If $\phi_{21} \neq 0$, the fact that the remainder of the first two rows vanishes implies that we can apply a $U(2)$ rotation to the first column, and rotate $\phi_{21}$ to 0 . Hence once again we end up with an element $\phi_{11}$ in an otherwise vanishing row and column. In either case the result is a disjoint $1 \times 1$ block matrix if $H(M)=U(M)$. Note that even though the matrix is the same if $H(M)=U S p(M)$, it is not necessarily disjoint, since this would require column 2 to vanish.

In the $O(N)$ case the argument is similar. Now we rotate the last $N-1$ rows so that the first column has one of the following three features:

$$
\phi_{31} \neq 0 ; \quad \phi_{21} \phi_{31}^{*}-\phi_{21}^{*} \phi_{31} \neq 0 .
$$

If the column group $H(M)$ is $U(M)$ these blocks are really disjoint, and we can repeat the process for the block matrix defined by the last $M-1$ column and the last $N-1$ or $N-2$ rows. This submatrix is treated in exactly the same way, and yields the same solutions. From the general argument in Sec. II C 5 we know that these solutions can only be combined with the upper-left block if they are identical, or 0 . This follows from the fact that their parameters $\rho_{p}$ are not proportional. The parameters are shown in Table II. We repeat this process until the remaining lower right block matrix is identically 0 .

If the column group is $U S p(M)$ we start in the same way. However, now the upper-left blocks are not strictly disjoint from the rest of the matrix, because $U S p(N)$ links columns 1 and 2. We can deal with the cases $G(N)=U(N)$ and $G(N)=O(N)$ simultaneously. The first step yields a $r \times 1$ upper-left block, where $r=1$ or 2 . Now we clean up column 2 using $G(N-r)$ rotations in the last $N-r$ rows. 
TABLE II. All solutions and the resulting subgroup embedding. In column $6, \omega_{5}=\lambda_{5} /\left|\lambda_{5}\right|$.

\begin{tabular}{|c|c|c|c|c|c|c|c|c|c|c|}
\hline Group & $P$ & $\rho_{2}$ & $\rho_{3}$ & $\rho_{4}$ & $\rho_{5}$ & $\mathbb{X}$ & $p$ & $q$ & Subgroup & Embedding \\
\hline$U(N) \times U(M)$ & 1 & 1 & $\cdots$ & $\cdots$ & $\cdots$ & A & 1 & 1 & $U(K)$ & $(0,0)$ \\
\hline$O(N) \times O(M)$ & 1 & 1 & $\ldots$ & $\ldots$ & $\ldots$ & A & 1 & 1 & $O(K)$ & $(0,0)$ \\
\hline$U S p(N) \times U S p(M)$ & 2 & 2 & $\ldots$ & $\ldots$ & $\cdots$ & $\mathrm{C}_{1}$ & 2 & 2 & $U S p(2 K)$ & $(0,0)$ \\
\hline$O(N) \times U(M)$ & 1 & 1 & $\ldots$ & 1 & $\ldots$ & A & 1 & 1 & $O(K)$ & $(0,1)$ \\
\hline$O(N) \times U(M)$ & 2 & 2 & $\cdots$ & 0 & $\cdots$ & B & 2 & 1 & $U(K)$ & $(3,0)$ \\
\hline$U(N) \times U S p(M)$ & 1 & 1 & 0 & $\ldots$ & .. & $\mathrm{A}_{0}$ & 1 & 2 & $U(K)$ & $(0,4)$ \\
\hline$U(N) \times U S p(M)$ & 2 & 1 & 1 & $\ldots$ & $\cdots$ & $\mathrm{C}_{1}$ & 2 & 2 & $U S p(2 K)$ & $(2,0)$ \\
\hline$O(N) \times U S p(M)$ & 1 & 1 & 0 & 1 & 0 & $\mathrm{~A}_{0}$ & 1 & 2 & $O(K)$ & $(0,5)$ \\
\hline$O(N) \times U S p(M)$ & 2 & 2 & 0 & 0 & 0 & $\mathrm{~B}_{0}$ & 2 & 2 & $U(K)$ & $(3,4)$ \\
\hline$O(N) \times U S p(M)$ & 2 & 1 & 1 & 1 & $\omega_{5}$ & $\mathrm{C}_{y}$ & 2 & 2 & $U(K)$ & $(3,4)$ \\
\hline$O(N) \times U S p(M)$ & 2 & 1 & 1 & 1 & $-\omega_{5}$ & $\mathrm{C}_{i y}$ & 2 & 2 & $U(K)$ & $(3,4)$ \\
\hline$O(N) \times U S p(M)$ & 4 & 2 & 2 & 0 & 0 & $\mathrm{D}$ & 4 & 2 & $U S p(2 K)$ & $(6,0)$ \\
\hline
\end{tabular}

Note that in those last $N-r$ rows all elements to the left of column 2 are 0 , and all elements to the right of column 2 are arbitrary complex numbers, so we can freely use $G(N-r)$ transformations acting on column 2 .

We get essentially the same three options as above. Option a is that column 2 vanishes completely. Then the upper block is disjoint in the $U S p(N)$ sense. Option b is that column 2 contains just one nonzero element, $\phi_{r+1,2}$. Then the homogeneous equation (11) tells us that the remainder of row 2 to the right of $\phi_{r+1,2}$ must vanish, and this makes the entire block disjoint in the $\operatorname{USp}(N)$ sense. Option $\mathrm{c}$ is that column 2 contains two nonvanishing elements. This can only happen if $G(N)=O(N)$ and if those two elements have different phases. Now we use both (11) and (13) [which indeed are available for $O(N)$ ] to show that rows $r+1$ and $r+2$ are 0 except on column 2 . We conclude that in all cases the $r \times s$ upper-left blocks are completely disjoint from the rest of the matrix. So now we can continue the argument in the last $N-r$ rows, and $N-s$ columns.

We have now reached a situation where both column 1 and 2 consist entirely of pivot elements, and we can apply the results of Sec. II C 3. This tells us that $\phi_{42}$ must be equal to $i \phi_{32}$ and that columns with one and two pivot elements cannot be combined. This leaves us with the following four possibilities for the upper-left block,

$$
\begin{aligned}
& \mathrm{A}_{0}:\left(\begin{array}{ll}
1 & 0
\end{array}\right) \quad \mathrm{C}_{x}:\left(\begin{array}{ll}
1 & 0 \\
0 & x
\end{array}\right) \\
& \mathrm{B}_{0}:\left(\begin{array}{ll}
1 & 0 \\
i & 0
\end{array}\right) \quad \mathrm{D}:\left(\begin{array}{ll}
1 & 0 \\
i & 0 \\
0 & x \\
0 & i x
\end{array}\right)
\end{aligned}
$$

Here $x$ is a phase, since we know from (14) that all columns must have equal norm. All four must be considered for $O(N) \times U S p(N)$ and only $A$ and $C$ for $U(N) \times U S p(N)$.
The value of $x$ requires some additional discussion. Consider first $\mathrm{C}_{x}$. If $G(N)=U(N)$ we can make $x$ real using a phase rotation, and then the equations of motion guarantee that $x=1$. But this is not true if $G(N)=O(N)$. In that case there is a $\lambda_{5}$ term in the potential, and the quantity $\rho_{5}$ exists, and is equal to $\left(x^{*}\right)^{2}$ [see (19)]. Then the condition that $\lambda_{5} \rho_{5}^{*}$ be real determines $x$ up to a factor $i$. The solutions are

$$
x=\sqrt{\frac{\lambda_{5}^{*}}{\left|\lambda_{5}\right|}} \equiv y ; \quad x=i \sqrt{\frac{\lambda_{5}^{*}}{\left|\lambda_{5}\right|}}=i y .
$$

In each case there are two roots, but using $O(N)$ we can change the sign of row 2, and map them to each other.

In case $\mathrm{D}$ we can combine an orthogonal $S O(2)$ rotation on the first two rows with a diagonal phase rotation on column 1 and 2 in $S U(2)$ to obtain

$$
\left(\begin{array}{cc}
(c+i s) e^{i \theta} & 0 \\
(-s+i c) e^{i \theta} & 0 \\
0 & x e^{-i \theta} \\
0 & i x e^{-i \theta}
\end{array}\right) .
$$

Now we can choose $\theta$ to cancel the phase of $x$, and choose $c$ and $s$ to cancel $\theta$ in $\chi_{11}$, so that the final result is

$$
D:\left(\begin{array}{cc}
1 & 0 \\
i & 0 \\
0 & 1 \\
0 & i
\end{array}\right)
$$

In Table II we summarize all solutions. The table is organized in terms of four vertical blocks that respectively specify the original intersecting brane group, the values of the parameter $P$ and $\rho_{p}$, the basic block matrix $\mathbb{X}$ and its size, and the unbroken subgroup and its embedding. The latter is discussed in the next section. Note that, as 
announced earlier, for a given combination of $G$ and $H$, there are no two cases with vectors $\rho_{p}$ that are proportional to each other. Therefore a general solution is a combination of $K$ identical basic blocks, and never a combination of different blocks.

Note that the subscript of the matrix $C$ denotes the value of $x$, but the subscripts on A and B have a different purpose: they indicate that the second column vanishes. This would be irrelevant if $H(m)=U(m)$, but it is needed in $U S p(M)$ in order for the block to be disjoint from the rest of the matrix.

\section{Subgroups}

Now we determine the subgroups that are left unbroken by these solutions. Since only identical blocks can be repeated, the general form of the vacuum is

$$
v \mathbb{1}_{K} \otimes \mathbb{X}
$$

where $\mathbb{X}$ denotes the blocks in the last column of the table and $\mathbb{1}_{K}$ is the $K \times K$ unit matrix.

We begin with a list of all subgroup embeddings that occur. First of all we need the "brane separation" embeddings

$$
\begin{aligned}
U(k+\ell) & \rightarrow U(k) \times U(\ell) \\
O(k+\ell) & \rightarrow O(k) \times O(\ell) \\
U S p(2 k+2 \ell) & \rightarrow U S p(2 k) \times U S p(2 \ell) .
\end{aligned}
$$

In all these cases the vector representation splits as $(V, 1)+(1, V)$. This embedding is used to split off the group $G(N-K p) \times H(M-K q)$ that acts trivially on the vacuum. This part of the breaking requires no further discussion, so we leave out the $G(N-K p) \times H(M-K q)$ factor of the unbroken subgroup henceforth.

Further discussion is needed to determine which parts of $G(K p) \times H(K q)$ survive, but roughly speaking it will be some diagonal subgroup of the two factors, obtained by means of a suitable left-right combination of one of the embeddings listed in Table III. By embedding 0 we mean the trivial one, available for $U, O$, and $U S p$. Embedding 1 is simply the restriction from complex matrices to real ones. Embedding 2 is the restriction from complex to quaternionic, i.e., unitary matrices are restricted to the subset

TABLE III. Basic subgroup embeddings.

\begin{tabular}{lccc}
\hline \hline Number & Group & Subgroup & Vector decomposition \\
\hline 0 & $G(p)$ & $G(p)$ & $\mathrm{V}$ \\
1 & $U(p)$ & $O(p)$ & $\mathrm{V}$ \\
2 & $U(2 p)$ & $U S p(2 p)$ & $\mathrm{V}$ \\
3 & $O(2 p)$ & $U(p)$ & $\mathrm{V}+\mathrm{V}^{*}$ \\
4 & $U S p(2 p)$ & $U(p)$ & $\mathrm{V}+\mathrm{V}^{*}$ \\
5 & $U S p(2 p)$ & $O(p)$ & $2 \mathrm{~V}$ \\
6 & $O(4 p)$ & $U S p(2 p)$ & $2 \mathrm{~V}$ \\
\hline \hline
\end{tabular}

$U^{*}=h U h^{T}$ where $h$ is antisymmetric. Embedding 3 is a well-known one, used in grand unified theory (GUT) theories for embedding $S U(5)$ GUTs in $S O(10)$; for further details see the Appendix. Embedding 4 is similar, and follows immediately from the standard basis used for symplectic groups, as explained in the Appendix. Embedding 5 is obtained by combining 4 and 1, and embedding 6 by combining 3 and 4 .

Embedding 3 is best understood by extending $O(2 K)$ to $U(2 K)$ and then conjugating the entire $O(2 K)$ group within $U(2 K)$. The resulting group matrices are not real, but this is as good a definition of $O(2 K)$ as the standard one. Moreover, we can always transform the result back to the real form, if we wish. For this transformation we use the matrix (A3), but with rows and columns rearranged into pairs, exactly as in the symplectic case (as explained in Appendix A 3). The matrix $Z$ now takes the form

$$
Z=\frac{1}{\sqrt{2}} \mathbb{1}_{K} \otimes\left(\begin{array}{cc}
1 & 1 \\
i & -i
\end{array}\right) .
$$

We transform the orthogonal group generators $O$ to $\tilde{O}=Z^{\dagger} O Z$, and multiply the vacuum matrix on the right by $Z^{\dagger}$. The advantage of this basis becomes clear when we make $O(N)$ act on vacua of the form B and $\mathrm{D}$, which have column vectors $(1, i)$. In the new basis these take the form $(1,0)$. There is a subgroup $U(K) \subset O(2 K)$ that acts on the odd indices as a unitary matrix $Y$, and on the even indices as $Y^{*}$. There are additional generators in $O(2 \mathrm{~K})$, but they map the odd components to the even ones, and this can never be repaired by a transformation acting on the columns.

The complexified $O(N)$ basis reveals some nice analogies between the symplectic and orthogonal cases, but it is probably not preferable to work in that basis from the start. First of all this basis is only useful for even $N$, and secondly the concept of disjoint matrices becomes less convenient in the complexified basis. Basis elements now come in pairs, as for $\operatorname{USp}(N)$, because the metric is build out of $2 \times 2 \sigma_{1}$ blocks. Hence only pairs can be disjoint. Another way of saying this is that the complexified basis is good for solutions of type B and D, but inconvenient for type A (as well as C).

We specify for each case the embedding of the subgroup in $G(K p) \times H(K q)$. This is done by specifying a pair of labels $(m, n)$ that each refer to a line in Table III. From $(m, n)$ one can determine the embedding in the vector representations of the two groups $G(K p)$ and $H(K q)$. From this we derive the decomposition of the Higgs field itself, which must include a singlet, corresponding to the vacuum expectation value (v.e.v.).

(i) $U(N) \times U(M)$, type $\mathbf{A}$. The vacuum, limited to the $K \times K$ subspace where the v.e.v. is nontrivial, is a multiple of the $K \times K$ unit matrix. This case was already discussed in [1]. We just use it to illustrate our notation. 


$$
\begin{aligned}
U(K) \times U(K) & \rightarrow U(K) \quad \text { embedding }(0,0) \\
(\mathrm{V}, \mathrm{V}) & \rightarrow 1+\operatorname{Adj}_{K} .
\end{aligned}
$$

Here $\operatorname{Adj}_{K}$ is the irreducible $U(K)$ representation of dimension $K^{2}-1$, the adjoint representation of $S U(K)$ subgroup of $U(K)$, with $U(1)$ charge 0 . The first component of the decomposition of the Higgs field, $(\mathrm{V}, \mathrm{V})$, is the singlet that corresponds to the Higgs v.e.v.

(ii) $O(N) \times O(M)$, type $\mathbf{A}$. This was also discussed in [1]. The result is

$$
\begin{aligned}
O(K) \times O(K) & \rightarrow O(K) \quad \text { embedding }(0,0) \\
(\mathrm{V}, \mathrm{V}) & \rightarrow 1+\mathrm{A}+\mathrm{S} .
\end{aligned}
$$

In this case the Higgs singlet comes out of the trace of the symmetric tensor.

(iii) $\operatorname{USp}(2 N) \times U S p(2 M)$, type $\mathbf{C}_{1}$. In terms of quaternions, the vacuum has the same form as the previous two examples. It is proportional to a $K \times K$ diagonal matrix of unit quaternions. The reason it appears here as type $C_{1}$ rather than $A$ is that we have written the quaternions in a complex base of twice the size. In terms of complex fields the vacuum is

$$
v \mathbb{1}_{K} \otimes\left(\begin{array}{ll}
1 & 0 \\
0 & 1
\end{array}\right) .
$$

Note that a diagonal with an odd number of nonvanishing entries does not even respect the quaternionic condition, so it cannot occur. The result is

$$
\begin{aligned}
& U S p(2 K) \times U S p(2 K) \rightarrow U S p(2 K) \\
& \text { embedding }(0,0) \\
&(\mathrm{V}, \mathrm{V}) \rightarrow 1+\mathrm{A}+\mathrm{S} .
\end{aligned}
$$

The only differences with the orthogonal case are that all dimensions are even, and that the antisymmetric representation is reducible: a symplectic trace must be removed. In the orthogonal case the symmetric representation is the one that must be made traceless. In both cases, the trace provides the Higgs representation.

(iv) $O(N) \times U(M)$, type $\mathbf{A}$. In this case the vacuum block matrix $\mathbb{X}$ is (1). This is very similar to the $U(N) \times U(M)$ and $U(N) \times U(M)$ breakings. Within the right $U(K)$ subgroup only the $O(K)$ transformations, the real subgroup of $O(K)$, can be compensated by orthogonal transformations. The remaining $U(K)$ transformations, acting infinitesimally, generate imaginary parts that cannot be removed by an orthogonal transformation. Therefore we get

$$
\begin{aligned}
O(K) \times U(K) & \rightarrow O(K) \quad \text { embedding }(0,1) \\
(\mathrm{V}, \mathrm{V}) & \rightarrow 1+\mathrm{S}+\mathrm{A} .
\end{aligned}
$$

(v) $O(N) \times U(M)$, type $\mathbf{B}$. If we use $K$ basic blocks B, then we get a vacuum matrix that can be brought into the form

$$
v \mathbb{1}_{K} \otimes\left(\begin{array}{l}
1 \\
i
\end{array}\right) .
$$

The part of $O(N)$ that is affected by the v.e.v. is $O(2 K)$. The determination of the symmetry of the vacuum can be done most efficiently by using the transformation (29). Clearly the left $U(K) \subset O(2 K)$ can be undone by a right $U(K)$ transformation. Hence the final result is

$$
\begin{aligned}
O(2 K) \times U(K) & \rightarrow U(K) \quad \text { embedding }(3,0) \\
(\mathrm{V}, \mathrm{V}) & \rightarrow 1+\operatorname{Adj}_{K}+\mathrm{A}+\mathrm{S} .
\end{aligned}
$$

(vi) $U(N) \times U S p(M)$, type $\mathbf{A}_{0}$. The block matrix $\mathbb{X}$ that defines the vacuum is

$$
\mathbb{X}=\left(\begin{array}{ll}
1 & 0
\end{array}\right) .
$$

With $K$ diagonal copies of that matrix, the effect is to break the $S U(2)$ factors acting on each column pair, so that only $U(K)$ remains. This combines with a $U(K)$ factor that acts on the row index. The result is

$$
\begin{aligned}
U(K) \times U S p(2 K) & \rightarrow U(K) \quad \text { embedding }(0,4) \\
(\mathrm{V}, \mathrm{V}) & \rightarrow 1+\operatorname{Adj}_{K}+\mathrm{A}+\mathrm{S} .
\end{aligned}
$$

Note that this is like the mirror image of case B for $O(N) \times U(M)$ discussed above, after using the $Z$ transformation (29).

(vii) $U(N) \times U S p(M)$, type $\mathbf{C}_{1}$. The block matrix $\mathbb{X}$ is the $2 \times 2$ unit matrix. Clearly, if we act on $K$ of these blocks with $U S p(2 K)$ from the left and the right, the diagonal combination is preserved. Hence we get

$$
\begin{aligned}
U(2 K) \times U S p(2 K) & \rightarrow U S p(2 K) \text { embedding }(2,0) \\
(\mathrm{V}, \mathrm{V}) & \rightarrow 1+\mathrm{A}+\mathrm{S} .
\end{aligned}
$$

(viii) $O(N) \times U S p(M)$, type $\mathbf{A}_{0}$. The vacuum is

$$
\mathbb{X}=\left(\begin{array}{ll}
1 & 0
\end{array}\right) .
$$

The affected part of the group is $O(K) \times U S p(2 K)$. The $O(K)$ subgroup of $O(N)$ can keep the vacuum invariant if it is combined with a $O(K)$ rotation of the $K 1 \times 2$ blocks. Hence we need to break $U S p(2 K)$ acting on those blocks first to $U(K)$ and then to $O(K)$. Hence we get

$$
\begin{aligned}
O(K) \times U S p(2 K) & \rightarrow O(K) \quad \text { embedding }(0,5) \\
(\mathrm{V}, \mathrm{V}) & \rightarrow 2 \times(1+\mathrm{A}+\mathrm{S}) .
\end{aligned}
$$

This subgroup $O(K) \subset U S p(2 K)$ is the maximal subgroup that can survive. The $S U(2)^{K}$ subgroups of $U S p(2 K)$ all change the vector $(1,0)$, and this can never be undone by a $O(K)$ transformation on the 
row indices. Likewise, the complex transformations in $U(K) \subset U S p(2 K)$ make the vacuum complex, and then an orthogonal transformation cannot make them real again.

(ix) $O(N) \times U S p(M)$, type $\mathbf{B}_{0}$. In this case the vacuum has the form

$$
v \mathbb{1}\left(\begin{array}{ll}
1 & 0 \\
i & 0
\end{array}\right) .
$$

The discussion is very similar to case B of $O(N) \times$ $U(M)$ combined with case A of $U(N) \times U S p(M)$. The surviving symmetry group is the diagonal combination of the $U(K)$ subgroup of $O(2 K)$ and the analogous $U(K)$ subgroup of $U S p(2 K)$.

$$
\begin{aligned}
O(2 K) \times U S p(2 K) \rightarrow & U(K) \quad \text { embedding }(3,4) \\
(V, V) \rightarrow & 2 \times\left(1+\operatorname{Adj}_{K}\right) \\
& +\mathrm{A}+\mathrm{S}+\mathrm{A}^{*}+\mathrm{S}^{*} .
\end{aligned}
$$

(x) $O(N) \times U S p(M)$, type $\mathbf{C}$. The matrix

$$
\left(\begin{array}{ll}
1 & 0 \\
0 & x
\end{array}\right)
$$

is in general complex, and cannot be made real by gauge transformations. Writing $x=e^{i \xi}$ we have

$$
\left(\begin{array}{cc}
1 & 0 \\
0 & e^{i \xi}
\end{array}\right) \simeq\left(\begin{array}{cc}
e^{i \xi / 2} & 0 \\
0 & e^{i \xi / 2}
\end{array}\right)=e^{i \xi / 2}\left(\begin{array}{ll}
1 & 0 \\
0 & 1
\end{array}\right),
$$

where $\simeq$ denotes gauge equivalence. The transformation used here is a diagonal $S U(2)$ transformation in $U S p(M)$. The overall phase $e^{i \xi / 2}$ cannot be transformed away. This is the only case among the six combinations of groups $\mathrm{U}, \mathrm{O}$, and $\mathrm{S}$ where such a phase can occur. Bifundamentals of type $(\mathrm{O}, \mathrm{O})$ and $(\mathrm{S}, \mathrm{S})$ are real or quaternionic, and such a phase violates these constraints; in any combination that involves a unitary group the phase can be gauged away. So only for $(\mathrm{O}, \mathrm{S})$ the phase exists and is not a pure gauge variable. This is also the reason for the existence of the $V_{5}$ terms in the potential. Without them, overall phase changes of the field $\phi_{i \alpha}$ would give rise to flat directions in the potential.

We may explore the potential along this phase direction. We get, keeping everything fixed except the phase,

$$
\begin{aligned}
V(\xi) & =\text { const }+\frac{1}{2} \lambda_{5} \operatorname{Re}^{2 i \xi}+\lambda_{5}^{*} \operatorname{Re}^{-2 i \xi} \\
& =\left|\lambda_{5}\right| R \cos \left(\eta_{5}+2 \xi\right),
\end{aligned}
$$

where $R$ is some real number and $\lambda_{5}=\left|\lambda_{5}\right| e^{i \eta_{5}}$. As a function of $0 \leq \xi<2 \pi$ the cosine has four extrema, at $\xi=-\frac{1}{2} \eta_{5}+\frac{1}{2} \ell \pi, \ell=0,1,2,3$. This implies

$$
x= \pm \sqrt{\frac{\lambda_{5}^{*}}{\left|\lambda_{5}\right|}} ; \quad x= \pm i \sqrt{\frac{\lambda_{5}^{*}}{\left|\lambda_{5}\right|}}
$$

as we have seen earlier. The signs can be gauged away, so that we end up with two distinct extrema, corresponding to the two options $\mathrm{C}_{x}$ and $\mathrm{C}_{i x}$ in Table II. Clearly, one of these extrema has more vacuum energy than the other and is therefore a saddle point. The lower of the two can be a saddle point of the full potential, or a local or global minimum, depending on the values of the other parameters $\lambda_{i}$.

In the minimal case, $N=M=2$, the matrix $\mathrm{C}_{1}$ breaks the group $O(2) \times U S p(2)$ to a diagonal $S O(2) \sim U(1)$, with the $S O(2)$ within $U S p(2) \equiv$ $S U(2)$ generated by $\sigma_{2}$ [the group is $S O(2)$ rather than $O(2)$ because $S U(2)$ does not contain $O(2)$ ]. If we replace $\mathrm{C}_{1}$ by $\mathrm{C}_{x}$, the $S O(2)$ generator is rotated within $S U(2)$ to $\cos (\xi) \sigma_{2}+\sin (\xi) \sigma_{1}$. Hence the $S O(2) \subset S U(2)$ embedding rotates inside $S U(2)$ as a function of the phase of $\lambda_{5}$. The final results for this embedding are, for both values of the complex parameter $x$,

$$
\begin{aligned}
O(2 K) \times U S p(2 K) \rightarrow & U(K) \quad \text { embedding }(3,4) \\
(V, V) \rightarrow & 2 \times\left(1+\operatorname{Adj}_{K}\right) \\
& +A+S+A^{*}+S^{*}
\end{aligned}
$$

Note that this is group theoretically the same embedding as in case $\mathbf{B}_{0}$. However, the subgroup is embedded in a different way in $U S p(N)$. This can most easily be clarified for the minimal case $N=M=2$. If we work with the real basis for $O(2)$ instead of the complexified basis used in the discussion of case $\mathbf{B}_{0}$, then on the $O(2)$ side the action is identical in both cases. There is only one generator, so we have no choice of embedding. However, in case $\mathrm{C}_{1}$ the $O(2)$ action is undone by an $O(2) \subset U S p(2) \sim S U(2)$ that is simply a real restriction of $S U(2)$, whereas in case $\mathbf{B}_{0}$ it is undone by an $S U(2)$ group element $e^{i \theta \sigma_{3}}$.

Although isomorphic subgroups are obtained, these are distinct vacua, with different vacuum energies.

(xi) $O(N) \times U S p(M)$, type $\mathbf{D}$. Now the vacuum is

$$
v \mathbb{1}_{K} \otimes\left(\begin{array}{cc}
1 & 0 \\
i & 0 \\
0 & 1 \\
0 & i
\end{array}\right) .
$$

We use the matrix (29) on the left, to bring the vacuum in the form

$$
\frac{v}{\sqrt{2}} \mathbb{1}_{K} \otimes\left(\begin{array}{cc}
1 & 0 \\
0 & 0 \\
0 & 1 \\
0 & 0
\end{array}\right) .
$$


On the left the canonical $U(2 K)$ subgroup of $O(4 K)$ is the only subset that has a chance to be compensated by a transformation from the right. But we do not have a full $U(2 K)$ available on the right; the maximal set of transformations is $\operatorname{USp}(2 K)$. Hence the left group must be broken one additional step further to $\operatorname{USp}(2 K)$. The final result is

$$
\begin{aligned}
O(4 K) \times U S p(2 K) & \rightarrow U S p(2 K) \text { embedding }(6,0) \\
(V, V) & \rightarrow 2 \times(1+A+S) .
\end{aligned}
$$

Note the similarity with case $\mathbf{A}_{0}$. Indeed, in all results there is a manifest similarity under exchange of orthogonal and symplectic transformations. This is also apparent in Table III.

\section{E. Comparison of vacuum energies}

Now we compare the vacuum energies of the solutions to determine the absolute minimum. This also provides insight about the reason all these solutions exist. The vacuum energy of a multiple solution built out of $K$ disjoint blocks with parameters $P$ and $Q$ is $-\frac{1}{2} K P \mu^{4} r^{2}$. This follows from Eq. (7) and the fact that all nonvanishing field values in a solution have the same absolute value. Their total number is the number of blocks, $K$, times the number of nonzero entries per block, $P$. This yields

$E(K, P, Q)=-\frac{K P \mu^{4}}{2\left(K P \lambda_{1}+Q\right)}=-\frac{\mu^{4}}{2\left(\lambda_{1}+Q / K P\right)}$.

The numerator must be positive for any allowed value of $Q$, $K$, and $P$. We see in the next section why this must be true, but it is already clear that a solution with negative numerator has positive vacuum energy, and hence can never be the absolute minimum. If the numerator is positive, the minimal energy is obtained for the minimal value of $Q / K P$.

For fixed $Q$ and $P$ this implies the following: If $Q<0$ the vacuum energy increases with $K$ so that the minimal energy is reached for the smallest nontrivial value of $K$, $K=1$. If $Q>0$ the vacuum energy decreases with $K$, and hence the minimum occurs for the largest value of $K$ that is allowed by $M$ and $N$, compared to the sizes $p$ and $q$ of the basic block given in Table II. To be precise

$$
K_{\max }=\min (\lfloor M / p\rfloor,\lfloor N / q\rfloor),
$$

where $\lfloor x\rfloor$ (the "floor function") is the largest integer smaller or equal to $x$. Only the values $K=1$ and $K=$ $K_{\max }$ can occur as absolute minima for suitable parameter values. This is in agreement with the results of [1]; in that case $Q=\lambda_{2}$.

Now we still have to compare different solutions. The ordering of solutions depends in a complicated way on the coupling constants, and it is not worthwhile to work this out in detail. But it is not difficult to see that-with one exception, see below-one can always make choices of $\lambda_{2} \ldots \lambda_{5}$ so that $Q$ is negative for one solution, and positive for all others. Then one can always make $\lambda_{1}$ large enough so that $\lambda_{1}+Q / K P>0$. The solution with negative $Q$ is then the global minimum. This implies that any solution in the table can be an absolute minimum for $K=1$.

The exception is one of the two solutions with block matrices $\mathrm{C}_{y}$ and $\mathrm{C}_{i y}$. Their values of $Q$, defined in (21), are respectively $Q_{y}=\lambda_{2}+\lambda_{3}+\lambda_{4}+\left|\lambda_{5}\right|$ and $Q_{i y}=\lambda_{2}+\lambda_{3}+$ $\lambda_{4}-\left|\lambda_{5}\right|$. Obviously $Q_{i y}<Q_{y}$, and hence only $Q_{i y}$ can be a global minimum, as we have seen already in a different way in the previous section.

The discussion for $K=K_{\max }$ is similar. For any solutionexcept the two just mentioned-there is a choice of coupling constants so that its value of $Q / P K_{\max }$ value is positive, but smaller than all others. Hence any of the solutions in the table, except $\mathrm{C}_{y}$, can occur as a global minimum for $K=K_{\max }$.

\section{F. Boundedness and existence of solutions}

Now we discuss two issues that are related: the fact that for certain parameters the potential becomes unbounded from below, and the existence of singularities in the set of solutions as a function of the couplings. The parameter $r$, defined by Eq. (20), must be real, and hence the argument of the square root must be non-negative. This implies that the quantity $\lambda_{1}+Q / K P$ must be non-negative. If the numerator is negative for just one solution, one might conclude that this merely implies that the corresponding solution does not exist, but we will see that in that case the potential is unbounded, so that the other solutions lose their physical relevance as well.

Consider first what happens if we vary $\lambda_{1}$, while keeping all other coupling constants fixed. For sufficiently large $\lambda_{1}$ the potential is bounded, and the quantities $\lambda_{1}+Q / K P$ are positive for all solutions. If we decrease $\lambda_{1}$ we reach a singularity at

$$
\lambda_{1}=-\frac{Q}{K P} .
$$

The first such singularity we encounter is the one with smallest value of $\frac{Q}{K P}$, which corresponds to the solution that is the global minimum. If we pass through the singularity, the vacuum energy jumps from $-\infty$ to $+\infty$, and $r$ becomes imaginary. Just before reaching the singularity the energy of the global minimum approaches $-\infty$, indicating that the potential has become unbounded. If we decrease $\lambda_{1}$ even more the potential remains unbounded, so that the set $\lambda_{1}+Q / K P=0$, a hyperplane in the space of all couplings, marks the separation between bounded and unbounded potentials.

This is illustrated in Fig. 1 for an example with a maximal $K$ of 8 . Here the $\lambda_{1} / \lambda_{2}$-plane is shown, and $Q=\rho_{2} \lambda_{2}+\Delta$. The parameter $\Delta$ is controlled by the remaining coupling constants, and in the plot we have 
chosen $\Delta$ to be positive. The lines intersect the $\lambda_{2}$ axis at $\lambda_{2}=-\Delta / \rho_{2}$. The grey zone is the region where the potential is bounded, for fixed $\lambda_{2}, \lambda_{4}$, and $\lambda_{5}$. Moving to the right along horizontal lines below the common intersection point one first encounters unbounded territory for $K=1$; above that point it happens for maximal $K$. This nicely illustrates how either the maximal or the minimal $K$ solution dominates. A classical solution defines a boundary line between bounded and unbounded regions if just to the right of that line the solution is a global minimum. Since all solutions with $K=1$ or $K=K_{\max }$ are the global minimum for suitable values of the couplings, all such solutions mark the boundary between bounded and unbounded somewhere in coupling space (as before, with the exception of $\mathrm{C}_{y}$ ).

The converse is also true. If one moves in coupling space from a bounded region to an unbounded region, there should be a classical solution corresponding to the boundary that separates the two regions. To see this more clearly, consider a situation where one coupling, $\lambda_{i}$, is just at the edge of stability for a value $\lambda_{i}=c$. Then if $\lambda_{i}$ is made slightly smaller still, $\lambda_{i}=c-\epsilon$, there is a direction in field space that is unbounded. We can consider a line trough field space in that field direction: a set of fields $\phi_{i \alpha}=t \xi_{i \alpha}$, so that the potential goes to $-\infty$ for $t \rightarrow \infty$. The potential along this direction is

$$
V(t)=-\mu^{2} t^{2} \rho_{\mu}+\frac{1}{2} \sum_{i} \lambda_{i} \rho_{i} t^{4}
$$

where $\rho_{\mu}$ and $\rho_{i}$ are some fixed numbers derived by plugging $\xi_{i \alpha}$ in the various terms in the potential (we use the same notation here as for the parameters $\rho_{i}$ characterizing solutions to the equations of motion, because this is just the generalization to general field values). As a function of $t$ this is a standard quartic potential, which we can analyze as a function of the coupling $\lambda=\sum_{i} \lambda_{i} \rho_{i}$. The minimum is at

$$
t^{2}=\frac{\mu^{2} \rho_{\mu}}{\lambda} ; \quad V_{\min }=-\frac{1}{2} \frac{\mu^{4} \rho_{\mu}^{2}}{\lambda} .
$$

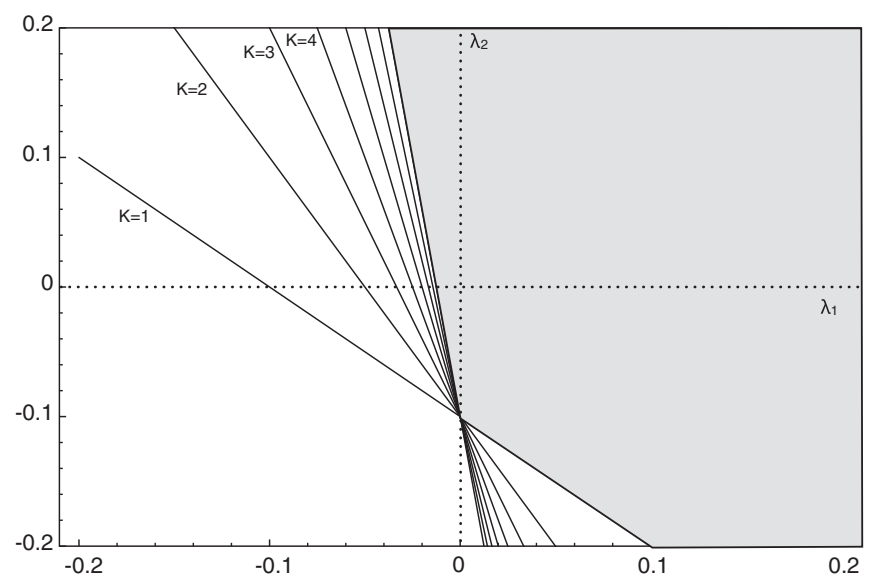

FIG. 1. Stability lines for $K \leq 4$. The grey area is the stable region.
Hence if $\lambda$ approaches 0 from the positive direction, the field goes to $\infty$ and the minimum to $-\infty$. On the other side of the stability line, for $\lambda_{i}=c+\epsilon$, the full potential is bounded from below, and its absolute minimum is a solution to the equations of motion. This absolute minimum may not coincide with the minimum along the aforementioned line, but it can only be lower than that. Hence it follows that there is a classical solution that becomes singular exactly at the boundary line.

This makes it immediately clear that if the potential has more terms, there must be more solutions. If we add another term to the potential, the plot acquires an extra dimension, and an additional hyperplane is needed to constrain the new coupling. This is true for all terms that are positive definite: $V_{1}, V_{2}, V_{3}$, and $V_{4}$. The corresponding coupling constants $\lambda_{1}, \lambda_{2}, \lambda_{3}$, and $\lambda_{4}$ are bounded from below, but not from above. But this is not true for $V_{5}$. This term is not positive definite: the value of $\lambda_{5} V_{5}+\lambda_{5}^{*} V_{5}^{*}$ can be sign flipped by replacing $\phi$ by $\sqrt{i} \phi$. Therefore $\left|\lambda_{5}\right|$ must be bounded. Projected on a real plane in coupling space this implies that $\lambda_{5}$ must be bounded from above and below. This explains the appearance of two additional solutions as soon as $\lambda_{5}$ is involved.

This is shown in Fig. 2, in the plane of $\lambda_{1}$ and the real part of $\lambda_{5}$. We have chosen $\lambda_{2}+\lambda_{3}+\lambda_{4}<0$, and these parameters are kept fixed. Furthermore $K_{\max }=4$. In this situation the stable region is bounded by four lines: the first solution with $K=1$, the first solution with $K=K_{\max }$, the second solution with $K=K_{\max }$, and the second solution with $K=1$. The terms first and second solution refer to the two straight lines that exist for every allowed value of $K$ in the real projection. In terms of complex $\lambda_{5}$, these lines become cones, and the two real solutions are connected by rotations in the complex plane. The solution with block matrix $\mathrm{C}_{i y}$ corresponds to the cone opening towards positive $\lambda_{1}$. This solution provides the boundary of the stability region. The other solution opens towards negative $\lambda_{1}$ and has the block matrix $\mathrm{C}_{y}$. It just provides local minima or saddle points.

\section{G. Bifundamentals: Summary}

In this section we considered Higgs symmetry breaking for gauge groups $G(N) \times H(M)$ with a bifundamental Higgs. The previous sections provide answers to the following questions:

(i) What are the stationary points in the potential, and to which subgroup does the group break in those points?

(ii) How do representations decompose under this breaking?

(iii) What is the global minimum?

Because of the length of this section we summarize here how one can obtain this information, without having to read all the arguments.

The answer to the first question is in Table II. To illustrate how this table is used, we give an example. Suppose one starts with a group $O(N) \times U(M)$. There are 


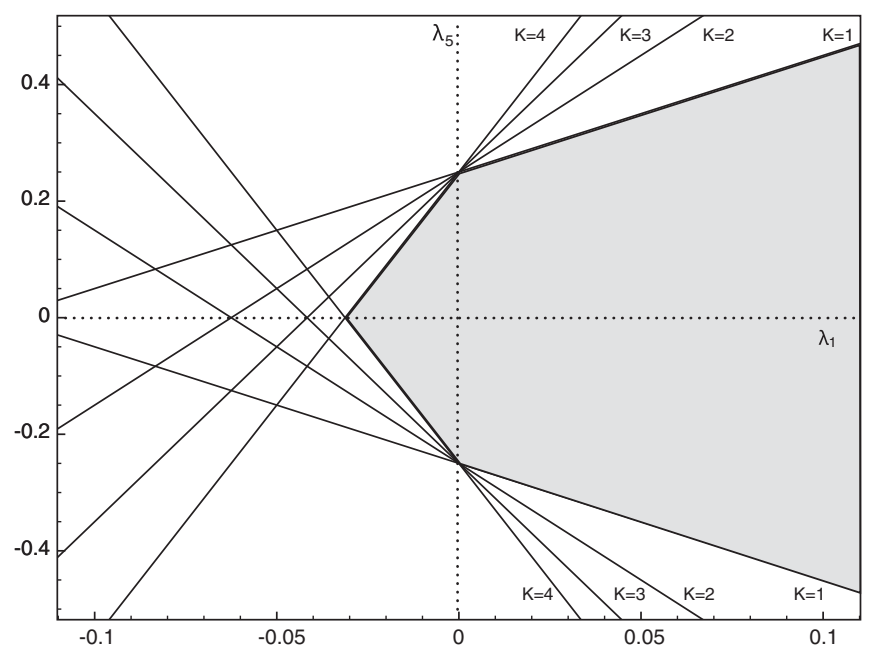

FIG. 2. Stability lines for $K \leq 4$ in the $\lambda_{1}, \lambda_{5}$ plane. The grey area is the stable region.

two lines in the table with that group in the first column, which means that there are two classes of minima that can occur in the potential. Suppose we take the second class, with $\mathbb{X}=B$. In columns 8 and 9 we find the number of rows $p$ and columns $q$ of $\mathbb{X}$. If we take $K$ diagonal copies of $\mathbb{X}$, there are $N-p K$ rows and $M-q K$ columns of the Higgs v.e.v. matrix that are 0 . Hence a subgroup $O(N-$ $p K) \subset O(N)$ and $U(M-q K) \subset U(M)$ remains unbroken. The remainder of these groups is then broken in such a way that the action of $O(p K)$ on the v.e.v. compensates that action of $U(q K)$. This combined action is specified in the column subgroup. The final result, in this particular example, is

$$
O(N) \times U(M) \rightarrow O(N-2 K) \times U(M-K) \times U(K) .
$$

The decomposition of representations $\left(R_{1}, R_{2}\right)$ of $O(N) \times$ $U(M)$ is obtained as follows. First one decomposes $O(N)$ to
$O(N-2 K) \times O(2 K)$ and $U(M)$ to $U(M-K) \times U(K)$. This is just the standard embedding (28), which can easily be applied to $R_{1}$ and $R_{2}$. Next one breaks the remainders of the left and right group to the common subgroup. In this example, that is the breaking $O(2 K) \times U(K)$ to $U(K)$. The embeddings in the left and right factor are specified in the last column in Table II, and the numbers in this column refer to the embeddings listed in Table III. In this example one needs on the left the nontrivial, but well-known embedding of $U(K) \subset O(2 K)$, whereas on the right the embedding is trivial, $U(K) \subset U(K)$. These embeddings must be applied to all the components of $R_{1}$ and $R_{2}$, and finally the resulting left and right representations are tensored in $O(N-2 K) \times$ $U(M-K) \times U(K)$.

What the global minimum is depends in a fairly complicated way on the relative values of the coupling constants, and we did not attempt to give exact analytical rules for that. It is much easier to determine that numerically using Eqs. (30) and (21). But there are two useful general statements. Only stationary points with $K=1$ or $K$ maximal can be the global minimum. If there is more than one class of solutions (i.e., more than one line in Table II), then each class can occur as the global minimum for appropriate choices of coupling constants, with the exception of the class with $\mathbb{X}=\mathrm{C}_{y}$.

\section{RANK-2 TENSORS}

In this section we deal with self-intersecting branes that give rise to rank-2 tensors. The allowed tensors are dependent on the allowed open string end points and on the symmetrization. Unitary branes allow two kinds of end points that are each other's conjugates; real and symplectic branes have only one. Furthermore one can in some cases remove traces to get irreducible representations. The possibilities are listed here.

$$
\begin{aligned}
& U(N) \text { Hermitian, traceless, complex } \quad \phi=\phi^{\dagger}, \quad \phi_{i}{ }^{i}=0 ; \quad \phi^{\prime}=U \phi U^{\dagger} \\
& U(N) \quad \text { Symmetric, complex } \quad \phi=\phi^{T} ; \quad \phi^{\prime}=U \phi U^{T} \\
& U(N) \quad \text { Antisymm. complex } \quad \phi=-\phi^{T} ; \quad \phi^{\prime}=U \phi U^{T} \\
& O(N) \quad \text { Symmetric, traceless, real } \quad \phi=\phi^{T}, \quad \phi_{i i}=0 ; \quad \phi^{\prime}=O \phi O^{T} \\
& O(N) \quad \text { Antisymm.real } \quad \phi=-\phi^{T} ; \quad \phi^{\prime}=O \phi O^{T} \\
& U S p(N) \quad \text { Symmetric, quaternionic } \quad \phi=\phi^{T}, \quad \phi^{\prime}=S \phi S^{T} \\
& U S p(N) \quad \text { Antisymm. quaternionic, traceless } \quad \phi=-\phi^{T} ; \quad \phi_{i j} \Omega_{i j}=0 ; \quad \phi^{\prime}=S \phi S^{T} .
\end{aligned}
$$

The matrices $U, O$, and $S$ are unitary, orthogonal (unitary and real) and symplectic (unitary and quaternionic), respectively. The matrix $\Omega$ is the symplectic metric, defined in Sec. II A 1.

\section{A. (Skew) diagonalization}

The last entry on each line shows the gauge transformation of the Higgs field $\phi$. A very useful fact is that in all these cases the matrix $\phi$ can be diagonalized or skew diagonalized by these transformations. This is well known for Hermitian matrices 
and unitary transformations, and for real symmetric matrices and orthogonal transformations. In both cases one gets a real diagonal matrix, with diagonal elements of either sign. Also well known is the fact that antisymmetric real matrices can be brought in skew-diagonal form using orthogonal transformations. This means that they consist of a diagonal of $2 \times 2$ blocks of the form

$$
a_{i}\left(\begin{array}{cc}
0 & 1 \\
-1 & 0
\end{array}\right)
$$

where $\lambda_{i}>0$ plus a number of vanishing $1 \times 1$ blocks. For quaternionic matrices subject to symplectic transformations essentially the same results hold as for real matrices and orthogonal transformations. If they are symmetric they can be diagonalized in terms of $2 \times 2$ blocks of the form [6]

$$
a_{i}\left(\begin{array}{ll}
1 & 0 \\
0 & 1
\end{array}\right),
$$

with $a_{i}$ being real, and if they are antisymmetric they can be skew diagonalized [7] in precisely the same form as the real antisymmetric matrices, using blocks (31). Finally, the result for symmetric complex matrices is somewhat less well known, and is called Autonne-Takagi factorization $[8,9]$. It is used in particle physics to deal with a Majorana mass matrix. This was not used in [1], even though these papers date back to the first quarter of last century. Instead, this was circumvented by considering the Hermitian combination $\phi \phi^{\dagger}$, which can be diagonalized in a more conventional way. The corresponding result for antisymmetric matrices was proved in [7]. It appears that some of these results have been rediscovered independently, and there may exist earlier references than the ones given here.

\section{B. The potential}

Much of the discussion here is similar to that for the $\mathrm{U}-\mathrm{U}, \mathrm{O}-\mathrm{O}$, and S-S bifundamentals. The requirement of having just a single field, with a single mass term, forces us to consider real field for $O(N)$ and quaternionic fields for $U S p(N)$, as was already assumed above. The quartic terms in the potential are precisely the same as in Eq. (2), with only the $\lambda_{1}$ and $\lambda_{2}$ terms. All remaining ones can be expressed in terms of the $\lambda_{2}$ term using the reality conditions. The only novelty is that in some cases there is a cubic term for Hermitian tensors of $U(N)$, symmetric tensors of $O(N)$, and antisymmetric tensors of $U S p(N)$. This happens for precisely the same fields that can have nontrivial traces.

A quick way of determining all terms is as follows. bifundamentals from different branes give rise to scalar fields $\phi_{i \alpha}$ with two distinct indices. Hence an invariant field combination, such as those appearing in the potential, has a matrix form consisting of a string of matrices where $\phi$ alternates with its transpose, which may either be $\phi^{T}$ or $\phi^{\dagger}$.
Every invariant must have one or more closed index loops. It follows that for bifundamentals every closed index loop must consist of an even number of matrices $\phi$.

Rank-2 tensors allow novel contractions between the two indices of $\phi$, because they now belong to the same group. But in addition such tensors always have a definite symmetry under transposition or Hermitian conjugation. The new options for index contractions may lead to new invariants, but every even index loop can always be brought to a form with alternating fields $\phi$ and $\phi^{T}$ or $\phi^{\dagger}$, and hence it is necessarily of a form we have already encountered for bifundamentals.

Therefore the only possible new terms must involve odd combinations of fields forming closed index loops. Denote such a combination as $(n)$. At second order terms of the form (2) and (1)(1) are possible, but since we wish to have only one massive propagating field we must set the combination (1), the trace, equal to 0 . Then at third order one can only have (3), and at fourth order there are no new terms at all [the first new term of even total order is (3)(3)].

A cubic term has the general form

$$
\kappa \phi_{i}{ }^{j} \phi_{j}{ }^{k} \phi_{k}{ }^{i},
$$

where the index is raised by a Kronecker $\delta$ or by $\Omega$ for the orthogonal and symplectic cases, respectively. In the Hermitian case the raised index distinguishes the action of $U$ from the action of $U^{*}$. Cubic terms do not exist for the (anti)-symmetric tensor of $U(N)$, because the index loop cannot be closed in an invariant way. They vanish for antisymmetric tensors of $O(N)$ and for symmetric symplectic tensors, because combining the field symmetries and the metric symmetries, they are found to be equal to minus themselves.

If there is neither a cubic term nor a tracelessness condition the discussion is similar to the one for bifundamentals. These two complicating factors are closely related: a trace may be thought of as a first-order interaction, and exists precisely when a cubic interaction exists. Indeed, a nontrivial trace can be dealt with by adding a linear term to the potential as a Lagrange multiplier, as was done in [1], and by treating its coupling as a degree of freedom that is varied.

\section{Cases without odd invariants}

Without these complications, the entire discussion in Sec. II C 5 applies, and we can view the solution as built out of the basic building blocks $\mathbb{X}=\mathbb{1}$ for the symmetric cases and $\mathbb{X}=i \sigma_{2}$ for the antisymmetric ones. Here $\mathbb{1}$ is $1 \times 1$ for $U(N)$ and $2 \times 2$ for $U S p(N)$. The equations of motion determining the eigenvalues $a_{i}$ are quadratic and identical for all $i$, but they only determine $a_{i}$ up to a sign.

It turns out that these signs can be rotated away in all cases. For the (anti)-symmetric unitary Higgs fields this is true because one can choose $U_{k l}=\delta_{k l} u_{k}$, with $u_{k}=i$ if $a_{k}$ 
is a negative eigenvalue. This is indeed precisely how one makes Majorana masses positive in the Lagrangian. It works only in $U(N)$, not in $S U(N)$. The sign of a symmetric $U S p(2 N)$ block $a_{i} \mathbb{1}$ can be flipped by means of the $S U(2)$ transformation $i \sigma_{3}$. The sign of a matrix $\mathbb{X}=$ $i \sigma_{2}$ can be flipped by $O(2)$ rotations $\sigma_{3}$ or $\sigma_{1}$ [note that this requires $O(2)$, and that it does not work in $S O(2)]$.

Since sign flips can be transformed away, this means that there is only one possible nonvanishing eigenvalue. Hence the most general solution consists of $K$ copies of that block. The energy of this solution is given by Eq. (30) with $P=1$ for $U(N)$ symmetric tensors, and $P=2$ in the other three cases. The parameter $\rho_{2}$ is equal to 1 in all cases (if the block matrix is $i \sigma_{2}$ the special form used in Sec. II C 4 cannot be obtained, but one can compute $\rho_{2}$ explicitly). Then we get

$$
\begin{array}{ll}
V=-\frac{K \mu^{4}}{2\left(K \lambda_{1}+\lambda_{2}\right)} & \text { for } U(N) \quad \text { (symmetric), } \\
V=-\frac{K \mu^{4}}{\left(2 K \lambda_{1}+\lambda_{2}\right)} & \text { for } U S p(N) \quad \text { (sym.); } \\
& U(N), O(N) \quad \text { (antisym.). }
\end{array}
$$

This agrees with [1] when comparable.

As before the unbroken symmetry groups fall into two classes: $K$ must be maximal if $\lambda_{2}>0$, and minimal for $\lambda_{2}<0$. The value of $\lambda_{1}$ is only relevant for stability of the potential: the denominators in (33) should always be positive. Although only the extreme cases, $K=1$ and $K$ maximal, can occur as absolute minima, all other values of $K$ are extrema (which may be local minima or saddle points). It is simplest to list the unbroken groups for all $K$,

$$
\begin{aligned}
U(N) & \rightarrow O(K) \times U(N-K) \quad \text { symmetric tensor } \\
U(N) & \rightarrow U S p(2 K) \times U(N-2 K) \quad \text { antisymmetric tensor } \\
O(N) & \rightarrow U(K) \times O(N-2 K) \quad \text { antisymmetric tensor } \\
U S p(N) & \rightarrow U(K) \times U S p(N-2 K) \quad \text { symmetric tensor. }
\end{aligned}
$$

The first two simply follow from the definition of orthogonal and symplectic groups as the invariance groups of a metric $h, U h U^{T}=h$, where $h$ is either the unit matrix or the antisymmetric unit matrix $\mathbb{1} \otimes i \sigma_{2}$ (see the Appendix). In the last two cases the subgroups are the simultaneous unitary invariance group of both a symmetric matrix $\mathbb{1} \otimes \sigma_{1}$ and the antisymmetric matrix $\mathbb{1} \otimes i \sigma_{2}$. One of these matrices defines the original unbroken gauge group, and the other is the Higgs v.e.v.

Writing the subgroups for all $K$ clarifies some special features of the special case $K=1$, especially regarding the global group. In particular: the symmetric tensor breaks
$U(N)$ to $U(N-1)$ times a $\mathbb{Z}_{2}$ symmetry, the $U(N)$ matrix $\operatorname{diag}(-1,1, \ldots, 1)$. This is $O(K)$ for $K=1$. The antisymmetric tensor breaks the first two components of $U(N)$ to $U S p(2)$. Without the result for all $K$ one might have called this $S U(2)$, which is correct, but gives the incorrect impression that special unitary groups appear after symmetry breaking. However, this happens only for the special unitary group $S U(2)$, which must be interpreted as $U S p(2)$. Apart from this isomorphism, we never get $S U(K)$ factors in the unbroken group. Finally in the third case the first factor is $U(1)$ and not $S O(2)$. These are isomorphic as groups, but using the correct notation avoids some subtle mistakes. First of all we see immediately that the first factor is $S O(2)$ and not $O(2)$, which is locally isomorphic to, but globally different from, $S O(2)$. Secondly, we never get special orthogonal groups, except $S O(2)$, because of the isomorphism with $U(1)$. This may not seem important, but the implication of not getting special unitary or orthogonal groups is that all broken subgroups can be realized in terms of membranes. We however do not explore this point further in this paper.

\section{Cases with odd invariants}

In this category we have three kinds of Higgs fields, Hermitian $U(N)$ tensors, real symmetric $O(N)$ tensor, and antisymmetric $U S p(N)$ tensors. A detailed analysis of the first two is in Appendix B of [1], and the only novelty here is the antisymmetric, symplectic case. However, in all three cases one ends up with the same equations, and hence the conclusions are also the same. We illustrate this for the antisymmetric tensor of $U S p(2 N)$, the only new case. The potential is

$$
\begin{aligned}
V= & -\mu^{2} \operatorname{Tr} \phi \phi^{\dagger}+\frac{1}{2} \lambda_{1}\left(\operatorname{Tr} \phi \phi^{\dagger}\right)^{2}+\frac{2}{3} \kappa \operatorname{Tr}(\phi \Omega)^{3} \\
& +\frac{1}{2} \lambda_{2} \operatorname{Tr}\left(\phi \phi^{\dagger}\right)^{2}-2 g \operatorname{Tr} \phi \Omega .
\end{aligned}
$$

The last term is a Lagrange multiplier; demanding stability with respect to $g$ variations yields the trace condition. We have included a factor of 2 for comparison with [1], because the rest of the potential also differs by a factor of 2. Substituting the skew-diagonal form (32) we get

$$
\begin{aligned}
V= & -2 \mu^{2} \sum_{i} a_{i}^{2}+2 \lambda_{1}\left[\sum_{i} a_{i}^{2}\right]^{2}+\frac{4}{3} \kappa \sum_{i} a_{i}^{3} \\
& +\lambda_{2} \sum_{i} a_{i}^{4}-4 g \sum_{i} a_{i} .
\end{aligned}
$$

The equations of motion for the eigenvalues $a_{i}$ are

$$
-\mu^{2} a_{i}+2 \lambda_{1} a_{i}\left[\sum_{j} a_{i}^{2}\right]+\kappa a_{i}^{2}+\lambda_{2} a_{i}^{3}-g=0 .
$$


TABLE IV. Rank-2 tensor breaking patterns.

\begin{tabular}{lcccc}
\hline \hline Group & $K$ & Symmetric tensor & Antisym. tensor & Adjoint \\
\hline$U(N)$ & 1 & $U(N-1) \times O(1)$ & $U(N-2) \times U S p(2)$ & $U(N-1) \times U(1)$ \\
$U(2 \ell)$ & $\operatorname{Max}$ & $O(2 \ell)$ & $U S p(2 \ell)$ & $U(\ell) \times U(\ell)$ \\
$U(2 \ell+1)$ & $\operatorname{Max}$ & $O(2 \ell+1)$ & $U S p(2 \ell) \times U(1)$ & $U(\ell) \times U(\ell+1)$ \\
$O(N)$ & 1 & $O(N-1) \times O(1)$ & $O(N-2) \times U(1)$ & $\ldots$ \\
$O(2 \ell)$ & $\operatorname{Max}$ & $O(\ell) \times O(\ell)$ & $U(\ell)$ & $\ldots$ \\
$O(2 \ell+1)$ & $\operatorname{Max}$ & $O(\ell) \times O(\ell+1)$ & $U(\ell) \times O(1)$ & $\ldots$ \\
$U S p(2 N)$ & 1 & $U S p(2 N-2) \times U(1)$ & $U S p(2 N-2) \times U S p(2)$ & $\ldots$ \\
$U S p(4 \ell)$ & $\operatorname{Max}$ & $U(2 \ell)$ & $U S p(2 \ell) \times U S p(2 \ell)$ & $\ldots$ \\
$U S p(4 \ell+2)$ & $\operatorname{Max}$ & $U(2 \ell+1)$ & $U S p(2 \ell) \times U S p(2 \ell+2)$ & $\ldots$ \\
\hline \hline
\end{tabular}

This is the same ${ }^{1}$ equation as (B18) of [1] apart from a factor of 2 in front of the second term. This factor is just the coefficient $P$ introduced in Sec. II C 4, which indeed is 2 for the basic $U S p(2 N)$ antisymmetric block, and 1 for the other two cases. Hence all results of [1] go though after a replacement of $\lambda_{1}$ with $2 \lambda_{1}$. This implies that two distinct absolute minima can exist, depending on $\lambda_{1}, \lambda_{2}$, and $\kappa$.

One may summarize all three cases at once: the rules and the dynamics are the same for Hermitian fields in $G(N)=$ $U(N)$, symmetric fields in $G(N)=O(N)$, and antisymmetric fields in $G(N)=U S p(2 N)$. In general, the group $G(N)$ splits into several components using the brane separation embedding (28). With some string theory intuition, this means that the stack of $N$ branes is split into several smaller stacks. There are stationary points where the group $G(N)$ splits into three parts, but for the global minima there are just two possible unbroken subgroups,

$$
G(N) \rightarrow G(N-1) \times G(1)
$$

and

$$
\begin{aligned}
G(N) & \rightarrow G\left(\frac{1}{2} N\right) \times G\left(\frac{1}{2} N\right) \quad(N \text { even }) \\
G(N) & \rightarrow G\left(\frac{1}{2}(N+1)\right) \times G\left(\frac{1}{2}(N-1)\right) \quad(N \quad \text { odd }) .
\end{aligned}
$$

According to [1], if $\kappa=0$ and $\lambda_{2}>0$ the second minimum is the lowest one, and when $\kappa$ is increased the first becomes the lowest one. If $\lambda_{2}<0$ we get the first minimum, and this remains true even if $\kappa$ is varied. Here $\lambda_{1}$ is assumed to be positive.

Note that in contrast to all other cases, the nontrivial part of the solution of the equations motion is not a combination of $K$ identical block matrices, but of two (or three, if we also count solutions that are not absolute minima) distinct $1 \times 1$ blocks. The reason why this happens can be understood by considering the weighted difference of two equations (35), with

\footnotetext{
${ }^{1}$ With $\kappa=\lambda_{3}$, and after correcting a typo in (B17).
}

variables $a_{1}$ and $a_{2}$. Multiplying the first with $a_{2}$, the second with $a_{1}$, and subtracting them we get

$$
\begin{aligned}
& \kappa a_{1} a_{2}\left(a_{1}-a_{2}\right)+\lambda_{2} a_{1} a_{2}\left(a_{1}-a_{2}\right)\left(a_{1}+a_{2}\right) \\
& \quad+g\left(a_{1}-a_{2}\right)=0 .
\end{aligned}
$$

In the absence of a cubic term and the Lagrange multiplier term, $\kappa=g=0$, this equation implies that the two eigenvalues must be the same or opposite, or one of them must vanish. Since in all relevant cases signs can be flipped, it follows that there can exist just one distinct nonvanishing eigenvalue. It is clear that either the existence of a cubic term or the tracelessness condition makes that argument invalid.

\section{Rank-2 tensors: Summary}

The main results of this section are given in Eqs. (34), (36), and (37). The embeddings used here are the same ones we already encountered for bifundamentals. In (34) one first applies the brane separation embedding (28) to split off the second factor on the right-hand side. Then the first factor is broken according to embeddings 1-4 of Table III for the four cases listed in Eq. (34), respectively. The embeddings in (36) and (37) are just brane separation embeddings (28); they are all of the form $G(N) \rightarrow G(N-K) \times G(K)$. All solutions come with an integer label $K$, but the global minimum only occurs for either $K=1$ or the maximal value of $K$ (depending on the coupling constants, as explained above). In the cases without odd invariants, the maximal value of $K$ is the one for which the second group factor in (34) is minimal or trivial. In the three cases with odd invariants, with $G(N) \rightarrow G(N-K) \times G(K)$, what we mean by maximal is the value where $G$ is maximally split, namely, $K=\lfloor N / 2\rfloor$ (note that the cases $K=k$ and $K=N-k$ are identical). We refer to this as $K=$ "max" in all cases.

In order to clarify the comparison with the results of [1] we have combined all the results for rank-2 tensors in Table IV, analogous to Table III of [1], but with rows and columns interchanged. The main differences with [1] are that we start with $U(N)$ as the unbroken group instead of 
$S U(N)$, and that we have included the results for symplectic groups. Furthermore we have left $O(1)$ factors that automatically appear if $K=1$ or $K=$ "max" is substituted in the general formula. They give rise to a $\mathbb{Z}_{2}$ discrete symmetry.

For $K=$ "max" one sometimes has to distinguish even and odd $N$, so we have used two separate lines in the table. Column 2 specifies $K$, and columns 3, 4, and 5 display the results for the various tensor representations of the Higgs field. Note that the adjoint representations of $O(N)$ and $U S p(N)$ are antisymmetric and symmetric tensors, respectively.

There are a few known errors in Table III of [1]. In [2] it was pointed out that a factor $U S p(2)$ was overlooked in the antisymmetric tensor breaking of $U(N)$ for $K=1$. Furthermore a $U(1)$ was overlooked in the adjoint breaking of $U(N)$ for $K=1$. There is one more error not mentioned in [2]: The antisymmetric tensor breaking of $U(N)$ for $K=$ "max" yields $U S p(2 \ell)$ and $U S p(2 \ell) \times U(1)$ for $N$ odd, and not $O(2 \ell+1)$ as stated in Table III of [1]. This is evidently just a transcription error in Table III, because in Sec. III C of [1] the correct result was given: $U(2 \ell) \rightarrow$ $S p(2 \ell)$ for $N=2 \ell$ and $S U(2 \ell+1) \rightarrow S p(2 \ell)$ for $N=2 \ell+1$, in agreement with our result [note the use of $S U(2 \ell+1)$ in [1] instead of $U(2 \ell+1)$ in our case].

\section{CONCLUSIONS}

The classic work of [1] from 1973 turns out to have an elegant generalization to all Higgs representations one can ever encounter in intersecting brane models. In all cases without trace conditions and cubic terms the solutions to the equations of motion are characterized by an integer $K$. The global minimum of the potential has $K$ either equal to 1 or the maximal value that can be realized. If the two intersecting brane groups are of different types, there are additional terms in the potential, and for each additional term there is an additional class of solutions. Each class is characterized by its own integer $K$. For suitable parameter values, each class can provide the absolute minimum, for either maximal or minimal $K$.

The foregoing holds for bifundamentals as well as selfintersections. However, in the latter case there are three cases with nontrivial traces and cubic terms, an orthogonal, a unitary, and a symplectic one. As already shown in [1] there are now more possibilities for extrema of the potential. They are not characterized by a single integer, but by two integers. In these extrema, the original group is split into various parts of the same type [i.e., products of $U\left(n_{i}\right), O\left(n_{i}\right)$, or $U S p\left(n_{i}\right)$ if the original group is $U(K), O(K)$, or $U S p(2 K)]$. The maximal number of parts one encounters is 3 , but in the absolute minimum the group is split into two parts only. Depending on the coupling constant values, it is either split into two equal (if $K$ is even) or almost equal parts (if $K$ is odd), or it is split in the smallest possible nontrivial part times the largest possible part.
It turns out that in all cases the unbroken subgroup can be written as a product of $U(K), O(K)$, and $U S p(2 K)$ factors. In particular, there are no special unitary or special orthogonal groups, except as a result of certain low-rank isomorphisms. This is likely to have a nice interpretation in terms of brane dynamics and the phenomenon known as brane recombination, but we leave further exploration of this point to future work, since here we only intended to address purely field-theoretic issues.

\section{ACKNOWLEDGMENTS}

It is a pleasure to thank Beatriz Gato-Rivera for discussions and contributions during an early stage of this work, and IFF-CSIC Madrid, where part of this work was done, for hospitality. This work was supported by the Netherlands Foundation for Fundamental Research of Matter (FOM) programme 156, "Higgs as Probe and Portal".

\section{APPENDIX: ORTHOGONAL AND SYMPLECTIC GROUPS}

Here we collect some facts about symplectic groups, and some related features of orthogonal groups acting on spaces of even dimension. Consider the subset of unitary $2 K \times 2 K$ matrices, $U(2 K)$, that satisfies the following restriction:

$$
U h U^{T}=h .
$$

For any $h$ this defines a subgroup of $U(2 K)$. Standard choices are $h_{S}=\mathbb{1}$, in which case the subgroup is $O(2 K)$, and the matrix

$$
h_{A}=\left(\begin{array}{cc}
0 & \mathbb{1} \\
-\mathbb{1} & 0
\end{array}\right)
$$

The resulting subgroup is called $U S p(2 K)$. In the definition of the orthogonal groups the restriction to even dimensions is not necessary, but the special features of interest here only hold in even dimensions.

To put the two groups on similar footing we may choose a different basis in the orthogonal case. In even dimensions we may choose instead of the metric $h_{S}=\mathbb{1}$ the symmetric matrix

$$
\tilde{h}_{S}=\left(\begin{array}{ll}
0 & \mathbb{1} \\
\mathbb{1} & 0
\end{array}\right)
$$

This defines a different subgroup of $U(2 N)$ that is isomorphic to $O(2 N)$. Their elements $\tilde{U}$ are related by a unitary matrix $Z$ in the following: $\tilde{U}=Z^{\dagger} U Z$. If $U h U^{T}=h$, then $\tilde{U} \tilde{h} U^{T}=\tilde{h}$, with $\tilde{h}=Z^{\dagger} h Z^{*}$. A useful choice is the matrix 


$$
Z=\frac{1}{\sqrt{2}}\left(\begin{array}{cc}
\mathbb{1} & \mathbb{1} \\
i \mathbb{1} & -i \mathbb{1}
\end{array}\right)
$$

then $h_{S}$ is transformed to $\tilde{h}_{S}$.

\section{The Lie algebra}

We work out the Lie algebra for a metric

$$
h=\left(\begin{array}{cc}
0 & \mathbb{1} \\
\epsilon \mathbb{1} & 0
\end{array}\right) .
$$

A generator $T$ of the Lie algebra is found to have the following form,

$$
T=\left(\begin{array}{cc}
H & S^{\dagger} \\
S & -H^{*}
\end{array}\right),
$$

where $H$ is Hermitian and $S$ is complex and satisfies

$$
S^{T}=-\epsilon S \text {. }
$$

The total number of real parameters of this Lie algebra is

$$
K^{2}+2 \times \frac{1}{2} K(K-\epsilon)=\frac{1}{2}(2 K)(2 K-\epsilon),
$$

which is indeed the correct answer for $O(2 K)(\epsilon=1)$ and $U S p(2 K)(\epsilon=-1)$.

The subset of generators with $S=0$ generates a $U(K)$ subalgebra, with corresponding group matrices

$$
U=\left(\begin{array}{cc}
Y & 0 \\
0 & Y^{*}
\end{array}\right)
$$

where $Y$ is unitary.

\section{Reality conditions}

Consider now any vector $\phi$ that $U$ acts on. If $U \in \mathcal{H}$ we can consistently limit the space on which it acts, in the following way:

$$
\phi=h \phi^{*}
$$

because

$$
U \phi=U h \phi^{*}=h U^{T-1} \phi^{*}=h U^{*} \phi^{*}=h(U \phi)^{*} .
$$

Hence the action of $\mathcal{H}$ preserves the condition $\phi=h \phi^{*}$. But we must also satisfy the consistency condition

$$
\phi=h \phi^{*}=h\left(h \phi^{*}\right)^{*}=h h^{*} \phi .
$$

Since this must hold for arbitrary vectors, this implies that $h h^{*}=1$. A symplectic (antisymmetric) metric does not satisfy this. Note that $h h^{*}=h\left(h^{\dagger}\right)^{T}$. If $h$ is antisymmetric, we get $-h h^{\dagger}$, which is negative definite. Hence we cannot impose such a condition on vectors. For an orthogonal group embedded with $h_{S}=\mathbb{1}$ this restricts $\phi$ to be real. If we use the symmetric metric $\tilde{h}_{S}$, the reality condition implies that $\phi$, written as a row vector, has the form $\left(\chi, \chi^{*}\right)$ where $\chi$ is a $K$-dimensional complex vector.

Although one cannot impose reality conditions on symplectic vectors, one can impose them on rank-2 tensors. Suppose a tensor $T$ transforms as $T \rightarrow U T V^{T}$, where $U$ and $V$ are symplectic matrices, not necessarily elements of the same group, and not necessarily of equal dimension. Suppose they satisfy $U h_{1} U^{T}=h_{1}$ and $V h_{2} V^{T}=h_{2}$. Now we can impose the condition

$$
T=h_{1} T^{*} h_{2}^{T}
$$

It is easy to check that this condition is preserved by the transformation. This condition can be imposed consistently if both matrices $h_{1}$ and $h_{2}$ are symmetric, or if both are antisymmetric. The former is simply the standard reality condition for orthogonal group tensor representations (if one uses $\left.h_{1}=\mathbb{1} \quad h_{2}=\mathbb{1}\right)$. We refer to the latter as a symplectic reality condition.

In some cases a basis exists where the symplectic reality condition becomes an ordinary reality condition. For example rank-2 tensor combinations of symplectic vectors are real representations, which means that such a basis does indeed exist. Similarly, the representation $(V, V)$ in $U S p(2) \times U S p(2)$ is real; it is the vector representation of $S O(4)$. This fact may or may not extend to $U S p(2 N) \times U S p(2 M)$, but in any case the real basis is not useful for our purposes, because it combines transformations of the two group factors.

\section{Quaternionic basis}

For both orthogonal and symplectic groups and algebras, there is another useful basis. It is obtained by reordering the original basis as $(1, K+1,2, K+2, \ldots, K-1,2 K)$. This splits the Lie-algebra matrices into $2 \times 2$ blocks. The matrices $h_{S}$ and $h_{A}$ take the form $\mathbb{1}_{K} \otimes \sigma_{1}$ and $\mathbb{1}_{K} \otimes i \sigma_{2}$, respectively. The block-diagonal symplectic transformations form a subgroup $S U(2)^{K}$, which is isomorphic to $U S p(2)^{K}$. The block-diagonal orthogonal transformations form a subgroup $U(1)^{K}$ or, equivalently, $S O(2)^{K}$.

We focus on the symplectic case. In the new basis the Lie-algebra generators can be written in terms of $2 \times 2$ blocks of the form

$$
\left(\begin{array}{cc}
a & b^{*} \\
b & -a^{*}
\end{array}\right)
$$


Hence they have four parameters each, except on the diagonal, where $a$ must be real.

The elements of the block matrix can be interpreted as quaternions. Quaternions are numbers of the form

$$
a+i b+j c+k d
$$

with $a, b, c, d \in \mathbb{R}$ and with $i^{2}=j^{2}=k^{2}=i j k=-1$. They can be represented by $2 \times 2$ matrices

$$
\left(\begin{array}{cc}
a+b i & c+d i \\
-c+d i & a-b i
\end{array}\right)
$$

This means that the multiplication of any two such matrices yields the answer representing the product of the corresponding quaternions. Note that the Lie-algebra generators are not precisely quaternions, but "imaginary" quaternions, quaternions multiplied by $i$. But the group elements are quaternions. This is analogous with orthogonal groups in a real basis: the group elements are real, and the Lie-algebra matrices are purely imaginary (in the standard physics convention).

Now consider a rank-2 tensor $T$ satisfying the symplectic reality condition (A9). We can write both $h_{1}$ and $h_{2}$ in blockdiagonal form. They are then equal to $h_{1}=\mathbb{1}_{M} \otimes\left(i \sigma_{2}\right)$ and $h_{2}=\mathbb{1}_{N} \otimes\left(i \sigma_{2}\right)$, where $M$ may be different from $N$. It is now easy to see that (A9) implies that the $2 \times 2$ blocks of $T$ are quaternions. Instead of (A9) one may define a reality condition with an opposite sign. If $T$ satisfies that condition, it is built out of quaternions times $i$. This is the analog of splitting a complex field in real and imaginary parts.

\section{Special forms of vectors}

It is well known that a real vector can be rotated into any direction using orthogonal rotations. This implies that any real vector can be orthogonally rotated to the form

$$
(r, 0, \ldots, 0) \text {, }
$$

where $r$ is real and positive. The same result holds for $U(N)$ acting on complex vectors. There is also an analogous result for symplectic transformations acting on quaternions. This can be seen as follows. Consider a quaternionic vector $\left(q_{1}, \ldots, q_{K}\right)$, where $q_{i}$ are quaternions. Up to normalization, quaternions are $S U(2)$ group elements. Therefore, using the $U S p(2 K)$ subgroup $S U(2)^{K}$ we can rotate all $q_{i}$ to the form $\left(d_{1} \mathbb{1}, \ldots, d_{K} \mathbb{1}\right)$, with $d_{i} \in \mathbb{R}$. Now we use the $U(K)$ subgroup of $U S p(2 K)$, or in fact just its $O(K)$ subgroup. This acts on $\left(d_{i}, \ldots, d_{k}\right)$ as a vector, and it can therefore rotate this vector so that only the first component is nonzero. Hence we get (A10) with $r$ interpreted as $r$ times a unit quaternion.

\section{a. Orthogonal and symplectic transformations of complex vectors}

In addition to this we also need such results for orthogonal and symplectic transformations acting on complex vectors. With orthogonal transformations acting on complex vectors we can first rotate all imaginary parts into the first entry, and then using $O(N-1)$ rotations on the last $N-1$ components rotate the remaining real components into the second entry. Then the simplified form is

$$
(x, r, 0, \ldots, 0) \text {, }
$$

with $x$ being complex and $r$ real. Note that $x$ must have an imaginary part; otherwise we can simplify further and rotate $r$ into $x$.

For symplectic transformations one can show that any vector can always be rotated to the form (A10), but this time with $r$ interpreted as a real number, and not as a quaternion. This is shown as follows. Using the $S U(2)^{K}$ subgroup we can rotate in each block the two-dimensional complex vectors to the form $(r, 0)$. This brings a general complex vector to the form $\left(r_{1}, 0, r_{2}, 0, \ldots, r_{K}, 0\right)$. Now, using $U(K)$ transformations, we can rotate away $r_{2}, \ldots r_{K}$. These $U(K)$ transformations also act on the odd components, but since they all vanish already this is irrelevant. It may seem that with $\operatorname{USp}(2 K)$ we can do the same as with $U(2 K)$, but this is not true. The difference with unitary rotations only becomes apparent when one tries to bring a second vector in a simplified form. The generic simplified form of two vectors in $U(2 K)$ is

$$
\left(\begin{array}{ccccc}
r_{1} & 0 & 0 & \ldots & 0 \\
x & r_{2} & 0 & \ldots & 0
\end{array}\right)
$$

where $x$ is a complex number. For $\operatorname{USp}(2 K)$ this is

$$
\left(\begin{array}{cccccc}
r_{1} & 0 & 0 & 0 & \ldots & 0 \\
x_{1} & x_{2} & r_{2} & 0 & \ldots & 0
\end{array}\right)
$$

since the first vector fixes rotations on the first two components, whereas in $U(2 K)$ it only fixes the first components. 
[1] L.-F. Li, Group theory of the spontaneously broken gauge symmetries, Phys. Rev. D 9, 1723 (1974).

[2] V. Elias, S. Eliezer, and A. R. Swift, Comment on Group theory of the spontaneously broken gauge symmetries, Phys. Rev. D 12, 3356 (1975).

[3] B. Gato-Rivera and A. N. Schellekens, GUTs without guts, Nucl. Phys. B883, 529 (2014).

[4] N. Marcus and A. Sagnotti, Group theory from quarks at the ends of strings, Phys. Lett. B 188, 58 (1987).

[5] D. Cremades, L. E. Ibanez, and F. Marchesano, Intersecting brane models of particle physics and the Higgs mechanism, J. High Energy Phys. 07 (2002) 022.
[6] F. Zhang, Quaternions and matrices of quaternions, Linear Algebra Appl. 251, 21 (1997).

[7] J. W. Stander and N. A. Wiegmann, Canonical forms for certain matrices under unitary congruence, Can. J. Math. 12, 438 (1960).

[8] L. C. Autonne, Sur les matrices hypohermitiennes et sur les matrices unitaires (J.-B. Baillière et fils, 1915).

[9] T. Takagi, On an algebraic problem related to an analytic theorem of Carathéodory and Fejér and on an allied theorem of Landau, Japanese Journal of mathematics 1, 83 (1924). 